\title{
NATURAL RESOLUTION OF ILL-POSEDNESS OF INVERSE KINEMATICS FOR REDUNDANT ROBOTS UNDER CONSTRAINTS*
}

\author{
S. ARIMOTO ${ }^{\dagger}$, J.-H. BAE ${ }^{\dagger}$, H. HASHIGUCHI ${ }^{\dagger}$, AND R. OZAWA ${ }^{\dagger}$ \\ Dedicated to Sanjoy Mitter on the occasion of his 70th birthday.
}

\begin{abstract}
In order to enhance dexterity in execution of robot tasks, a redundant number of degrees-of-freedom (DOF) is adopted for design of robotic mechanisms like robot arms and multifingered robot hands. Associated with such redundancy in DOFs relative to the number of physical variables necessary and sufficient for description of a given task, an extra performance index is introduced for controlling such a redundant robot in order to avoid arising of ill-posedness of inverse kinematics from the task space to the joint space. This paper shows that such an ill-posedness problem of DOF redundancy can be resolved in a natural way on the basis of construction of sensory feedback signals from the task space and a novel concept named "stability on a manifold". To show this, two illustrative robot tasks are analyzed in details, which are 1) posture control of an object via rolling contact by a redundant multi-DOF finger and 2) stable pinching and object manipulation by a pair of multi-DOF robot fingers.
\end{abstract}

Keywords: Redundancy Resolution, Robot Task, Redundant Robot, Stability on a Manifold, Constraint Manifold

1. Introduction. If a robot is designed so as to mimic human limb then its mechanism must be kinematically redundant, that is, its total degrees of freedom is larger than the number of independent physical variables required for description of a given motion task. This kinematic redundancy contributes to enhancement of dexterity and versatility in execution of robot tasks as discussed in a variety of literatures and books [1]-[7]. However, such redundancy of DOFs usually increases the complexity of robot dynamics and therefore makes control problems for execution of given tasks more difficult. It is emphasized in particular that in such a case the inverse kinematics from the operational space (task-description space) to the robot joint space becomes ill-posed. In order to avoid this ill-posedness, many methods have been proposed, most of which are based on an idea of introducing some extra performance criterion for determining uniquely an appropriate joint space trajectory by minimizing the criterion. This paper proposes a novel method for resolving such an ill-posedness problem related to redundancy of DOFs by a natural way without introducing any extra performance criterion. Instead, a novel concept named "stability on a manifold" is introduced [8]-[10] and it is shown that there exists a sensory feedback based on measurements of physical variables of task description so that it

\footnotetext{
*Accepted for publication on February 27, 2004.

${ }^{\dagger}$ Department of Robotics, Ritsumeikan University, 1-1-1 Nojihigashi, Kusatsu, Shiga, 525-8577
} Japan. 
enables the state of the overall system naturally and coordinately to converge to a lower-dimensional constraint manifold corresponding to a set of states fulfilling a target given task. The original idea of "stability on a manifold" was first introduced in control of multi-fingered hands for stable grasping and object-manipulation [8][10]. If an objective system under constraint is not redundant in DOF, then the concept of stability on a manifold is reduced to the conventional meaning (in the sense of Lyapunov) of stability for descriptor systems or mechanical systems under constraints, which was treated by Müller [11] [12] and Bajić [13]. However in the case of redundant mechanical dynamic systems under constraints as treated in this paper, the set of all still states corresponding to necessary and sufficient description of objective tasks is not a single state of zero-dimension but constitutes a non-zero dimensional manifold. In this paper, two typical robotic tasks are analyzed, which are 1) posture control of an object via rolling contact by a multi-DOF finger and 2) stable pinching and object manipulation by a pair of multi-DOF robot fingers. It is shown that in both cases proposed feedback control signals are of a simpler form than those obtained by conventional methods of using extra performance criteria and render in a natural way solution trajectories of the closed-loop dynamics convergent to each lower-dimensional manifold corresponding to target tasks without solving the problem of inverse kinematics. That is, seeking for some inverse kinematics is not indispensable in principle.

\section{Dynamics of Control of a Pivoted Object by a Robot Finger with} Redundant DOFs. Consider a problem of posture control of a pivoted object by means of a redundant multi-DOF robot finger as shown in Fig. 1. The object with a flat surface is pivoted at the fixed point $O_{m}\left(x_{m}, y_{m}\right)$ and hence only rotational motion around $O_{m}$ in the $x y$-plane is permitted. The problem is to control the rotational angle $\theta$ toward the desired value $\theta_{d}$ by a 3 -DOF planar robot finger. Hence the overall

motion of both the robot and the object is confined to the $x y$-plane and the gravity force can be ignored. Then, the kinetic energy of the system can be expressed as

$$
K=\frac{1}{2} \dot{q}^{\mathrm{T}} H(q) \dot{q}+\frac{1}{2} I \dot{\theta}^{2},
$$

where $q=\left(q_{1}, q_{2}, q_{3}\right)^{\mathrm{T}}, H(q)$ and $I$ denote the inertia matrix of the finger and the inertia moment of the object around $O_{m}$. Since the finger-end hemisphere contacts with the surface of the object, the following constraint equation follows:

$$
Q=-(r+l)+\left(x_{m}-x_{0}\right) \cos \theta-\left(y_{m}-y_{0}\right) \sin \theta=0 .
$$

On the other hand, the rolling contact without slipping induces the constraint that two speeds of the contact point $O_{1}\left(x_{1}, y_{1}\right)$ relative to $\phi r$ and $Y$ must be coincident, that is,

$$
\frac{\mathrm{d}}{\mathrm{d} t}(\phi r)=-\frac{\mathrm{d}}{\mathrm{d} t} Y
$$


where

$$
\left\{\begin{array}{l}
Y=\left(x_{0}-x_{m}\right) \sin \theta+\left(y_{0}-y_{m}\right) \cos \theta \\
\phi=\pi+\theta-q_{1}-q_{2}-q_{3}=\pi+\theta-q^{\mathrm{T}} e
\end{array}\right.
$$

with $e=(1,1,1)^{\mathrm{T}}$. Since eq.(3) can be easily integrated in such a way that

$$
R=-c_{0}+Y+\phi r=0,
$$

where $c_{0}$ is an integral constant, it is possible to write the Lagrangian by introducing Lagrange multipliers $f$ and $\lambda$ correspondingly to the quantities $Q$ and $R$ in the following way:

$$
L=K+f Q+\lambda R .
$$

Thus, by applying Hamilton's principle to the Lagrangian it is possible to obtain Lagrange's equation of motion for this system, which is described as follows:

$$
\begin{gathered}
\left\{H(q) \frac{\mathrm{d}}{\mathrm{d} t}+\frac{1}{2} \dot{H}(q)+S(q, \dot{q})\right\} \dot{q}-\left(\frac{\partial Q}{\partial q}\right)^{\mathrm{T}} f-\left(\frac{\partial R}{\partial q}\right)^{\mathrm{T}} \lambda=u, \\
I \ddot{\theta}-\frac{\partial Q}{\partial \theta} f-\frac{\partial R}{\partial \theta} \lambda=0 .
\end{gathered}
$$

More in details, Jacobian vectors in eqs.(7) and (8) can be calculated as

$$
\left\{\begin{array}{l}
\partial Q / \partial q=-(\cos \theta,-\sin \theta) J_{0}(q) \\
\partial R / \partial q=(\sin \theta, \cos \theta) J_{0}(q)-r e^{\mathrm{T}} \\
\partial Q / \partial \theta=Y, \quad \partial R / \partial \theta=-l,
\end{array}\right.
$$

where $J_{0}(q)=\partial\left(x_{0}, y_{0}\right)^{\mathrm{T}} / \partial\left(q_{1}, q_{2}, q_{3}\right)$, the Jacobian matrix of $\left(x_{0}, y_{0}\right)^{\mathrm{T}}$ (the cartesian coordinates of the center of curvature $O_{0}$ of the finger-end hemisphere) with respect to the joint coordinates $\left(q_{1}, q_{2}, q_{3}\right)$. Hence, the equation of motion of the object can be written in details as follows:

$$
I \ddot{\theta}-Y f+l \lambda=0 .
$$

Now, consider the control problem of maneuvering the object toward a specified rotational angle $\theta_{d}$ with a desired pushing force $f_{d}$. If a vision sensor can detect the rotation angle $\theta(t)$ of the object, it is possible to define a control signal described as

$$
u=-C \dot{q}-\left(\frac{\partial Q}{\partial q}\right)^{\mathrm{T}} f_{d}-f_{d} Y e-\left(\frac{\partial R}{\partial q}\right)^{\mathrm{T}}(\beta \Delta \theta+\alpha \dot{\theta}),
$$

where $\Delta \theta=\theta-\theta_{d}$. Substituting this control into eq.(7) yields the closed-loop dynamics

$$
\begin{aligned}
& \left\{H(q) \frac{\mathrm{d}}{\mathrm{d} t}+\frac{1}{2} \dot{H}(q)+S(q, \dot{q})+C\right\} \dot{q} \\
& -\left(\frac{\partial Q}{\partial q}\right)^{\mathrm{T}} \Delta f-\left(\frac{\partial R}{\partial q}\right)^{\mathrm{T}}(\lambda-\beta \Delta \theta-\alpha \dot{\theta})+Y f_{d} e=0 .
\end{aligned}
$$


Although the object dynamics expressed by eq.(10) do not change because they do not have any direct control input, but it is convenient to rewrite them in the following way:

$$
I \ddot{\theta}-Y \Delta f+l(\lambda-\beta \Delta \theta-\alpha l \dot{\theta})-Y f_{d}+\beta l \Delta \theta+\alpha l \dot{\theta}=0
$$

Taking inner product between $\dot{q}$ and eq.(12), multiplying eq.(13) with $\dot{\theta}$, and summing these resultant quantities, we obtain

$$
\left\{\begin{array}{l}
\frac{\mathrm{d}}{\mathrm{d} t} V=-\dot{q}^{\mathrm{T}} C \dot{q}-\alpha l \dot{\theta}^{2} \\
V=\frac{1}{2}\left\{\dot{q}^{\mathrm{T}} H(q) \dot{q}+I \dot{\theta}^{2}+\beta l \Delta \theta^{2}+\frac{f_{d}}{r} Y^{2}\right\} .
\end{array}\right.
$$

The scalar quantity $V$ is not positive definite in the eight-dimensional state space $(q, \theta, \dot{q}, \dot{\theta})$ but positive definite in the four-dimensional constraint manifold

$$
M_{4}=\left\{\left(q^{\mathrm{T}}, \theta, \dot{q}^{\mathrm{T}}, \dot{\theta}\right)^{\mathrm{T}}: Q=0, R=0, \dot{Q}=0, \dot{R}=0\right\}
$$

because $V$ includes quadratic terms of two positional variables $\Delta \theta$ and $Y$. Hence, the well-known theorem of LaSalle can be applied to eq.(14). However, in the case that the control objective is only to stop motion of the object by using a feedback signal

$$
u=-C \dot{q}-\left(\frac{\partial Q}{\partial q}\right)^{\mathrm{T}} f_{d}-f_{d} Y e
$$

the scalar function $V$ becomes of the form

$$
V_{0}=\frac{1}{2}\left\{\dot{q}^{\mathrm{T}} H(q) \dot{q}+I \dot{\theta}^{2}+\frac{f_{d}}{r} Y^{2}\right\} .
$$

This quantity is no more positive definite in the constraint manifold $M_{4}$ defined by eq.(15).

To simplify the terminologies in the following sections, we use symbols $\boldsymbol{x}=$ $\left(q^{\mathrm{T}}, \theta\right)^{\mathrm{T}}$ and $\dot{\boldsymbol{x}}=\left(\dot{q}^{\mathrm{T}}, \dot{\theta}\right)^{\mathrm{T}}$ and introduce a one-dimensional manifold $M_{1}$ defined as

$$
M_{1}=\{(\boldsymbol{x}, \dot{\boldsymbol{x}}=0): \dot{\boldsymbol{x}}=0, Q=0, R=0, Y=0\} .
$$

It is further convenient to introduce a neighborhood $N^{8}(r)$ with radius $r$ of a given still state $\boldsymbol{x}^{0}$ on $M_{1}$ in such a manner that

$$
N^{8}(r)=\left\{(\boldsymbol{x}, \dot{\boldsymbol{x}}): \frac{1}{2} \dot{q}^{\mathrm{T}} H(q) \dot{q}+\frac{I}{2} \dot{\theta}^{2}+\frac{1}{2} \Delta q^{\mathrm{T}} H(q) \Delta q+\frac{I}{2}\left(\theta-\theta^{0}\right)^{2} \leq r^{2}\right\},
$$

where $\Delta q=q-q^{0}$ and $\boldsymbol{x}^{0}=\left(q^{0}, \theta^{0}, \dot{q}=0, \dot{\theta}=0\right)$. Further, we assume that for the given still state $\boldsymbol{x}^{0}$ on $M_{1}$ there exists a positive number $r_{0}$ such that at any state $(\boldsymbol{x}, \dot{\boldsymbol{x}})$ in $N^{8}\left(r_{0}\right)$ the $2 \times 4$ matrix

$$
J=\left(\left(\frac{\partial R}{\partial \boldsymbol{x}}\right)^{\mathrm{T}},\left(\frac{\partial Q}{\partial \boldsymbol{x}}\right)^{\mathrm{T}}\right)^{\mathrm{T}}
$$

is of full rank (non-degenerated). 
3. Stability on a Manifold and Natural Redundancy Resolution. In the case of control for stopping the rotational movement of the object in Fig. 1, a target state can be fully described by specifying $\dot{\boldsymbol{x}}=0$ and $Y=0$, which means that the target state must lie on $M_{1}$ but the values for other variables $\theta$ and $q_{i}(i=1,2,3)$ need not be specified unless contact between the finger end and the object is broken and the whole state $(\boldsymbol{x}(t), \dot{\boldsymbol{x}}(t))$ for any $t>0$ remains in $N^{8}\left(r_{0}\right)$ where the Jacobian matrix $J(\boldsymbol{x})$ is non-degenerated. Usually, since the inverse kinematics from the task description ( $\dot{\boldsymbol{x}}=0, Q=0, R=0, Y=0$ ) to the joint coordinates is ill-posed because there are many states $(\boldsymbol{x}, \dot{\boldsymbol{x}})$ satisfying the task which are lying on $M_{1}$, the previous researches [1]-[7] proposed a variety of methods for resolving this ill-posedness of joint redundancy by introducing some appropriate extra performance index so that optimization of the index leads to unique determination of the inverse joint state. However, in this paper, it is shown that such an ill-posedness problem of inverse kinematics can be resolved in a natural way by introducing a concept of "Stability on a Manifold" as shown in the case of control problems for grasp and object manipulation of multi-fingered hands [8]-[10]. To do this, it is necessary to define another concept of neighborhoods of the reference still state $\boldsymbol{x}^{0} \in M_{1}$ on the manifold $M_{4}$ in the following way:

$$
N_{4}(\varepsilon)=\left\{(\boldsymbol{x}, \dot{\boldsymbol{x}}): Q=0, R=0, \dot{Q}=0, \dot{R}=0, V_{0} \leq \varepsilon^{2}\right\} .
$$

We are now in a position to introduce the definitions of stability on a manifold and asymptotic transferability to a manifold as follows:

Definition. (Stability on a manifold) If for any $\varepsilon>0$ there exist $\delta(\varepsilon)>0$ depending on $\varepsilon>0$ and another constant $r_{1}>0$ being less than $r_{0}$ and independent of $\varepsilon$ such that any solution to eqs.(12) and (13) with $\beta=0$ and $\alpha=0$ starting from an arbitrary initial state lying on $N_{4}(\delta(\varepsilon)) \cap N^{8}\left(r_{1}\right)$ remains in $N_{4}(\varepsilon) \cap N^{8}\left(r_{0}\right)$ for any $t>0$, then the reference still state $\left(\boldsymbol{x}^{0}, \dot{\boldsymbol{x}}=0\right)$ is said to be stable on a manifold (see Fig. 2).

Definition. (Asymptotic transferability to a manifold) If there exist positive values $\delta_{1}>0$ and $r_{1}>0$ (less than $r_{0}$ ) such that any solution starting from an arbitrary initial state in $N_{4}\left(\delta_{1}\right) \cap N^{8}\left(r_{1}\right)$ remains in $M_{4} \cap N^{8}\left(r_{0}\right)$ and converges asymptotically to some state in the set $M_{1} \cap N^{8}\left(r_{0}\right)$ as $t \rightarrow \infty$, then the neighborhood $N_{4}\left(\delta_{1}\right) \cap N^{8}\left(r_{1}\right)$ together with the reference state $\left(\boldsymbol{x}^{0}, 0\right)$ is asymptotically transferable to $M_{1} \cap N^{8}\left(r_{0}\right)$ (see Fig. 3).

The principal purpose of the paper is to show that a reference still state $\left(\boldsymbol{x}^{0}, 0\right)$ of the closed-loop equations of eqs.(12) and (13) with $\beta=0$ and $\alpha=0$ is stable on a manifold under the condition that in a neighborhood of the reference state $\left(\boldsymbol{x}^{0}, 0\right)$ the following $3 \times 4$ matrix $A$ is of full-rank (non-degenerated)

$$
A=\left(\left(\frac{\partial R}{\partial \boldsymbol{x}}\right)^{\mathrm{T}},\left(\frac{\partial Q}{\partial \boldsymbol{x}}\right)^{\mathrm{T}},-\boldsymbol{e}\right)^{\mathrm{T}}
$$


where $\boldsymbol{e}=\left(e^{\mathrm{T}},-1\right)^{\mathrm{T}}=(1,1,1,-1)^{\mathrm{T}}$. The necessity of this condition can be easily understood since the closed-loop dynamics of eqs.(12) and (13) with $\beta=0$ and $\alpha=0$ when the feedback signal of eq.(16) is used can be expressed as

$$
\bar{H}(q) \ddot{\boldsymbol{x}}+\left\{\frac{1}{2} \dot{\bar{H}}(q)+\bar{S}(q, \dot{q})+\bar{C}\right\} \dot{\boldsymbol{x}}-A^{\mathrm{T}} \boldsymbol{\lambda}=0
$$

where

$$
\left\{\begin{array}{l}
\boldsymbol{\lambda}=\left(\Delta f, \lambda, f_{d} Y\right) \\
\bar{H}(q)=\left(\begin{array}{cc}
H(q) & 0 \\
0 & I
\end{array}\right), \bar{S}=\left(\begin{array}{cc}
S(q, \dot{q}) & 0 \\
0 & 0
\end{array}\right), \bar{C}=\left(\begin{array}{cc}
C & 0 \\
0 & 0
\end{array}\right) .
\end{array}\right.
$$

It is evident from eq.(23) that if $\dot{\boldsymbol{x}}$ and $\ddot{\boldsymbol{x}}$ tend to zero as $t \rightarrow \infty$ then the nondegenerated property of $A$ implies $\lambda \rightarrow 0$ as $t \rightarrow \infty$. Since control of the object can be started from ordinary initial position as shown in Fig. 1, it is reasonable to assume that R1) $\left.\pi / 4<q_{1}<\pi,-\pi / 2 \leq q_{2}, q_{3} \leq \pi / 2,|\theta| \leq \pi / 4, \mathrm{R} 2\right)\left(x_{0}(q)-\right.$ $\left.\left.x_{m}\right)^{2}+\left(y_{0}(q)-y_{m}\right)^{2}<(r+l)^{2}+r^{2}, \mathrm{R} 3\right) l_{3}+\sqrt{(r+l)^{2}+r^{2}}<x_{m}, y_{m}$, and R4) $\left(l_{1}+l_{2}+l_{3}\right)>\sqrt{x_{m}^{2}+y_{m}^{2}}+\sqrt{(r+l)^{2}+r^{2}}$. Under this condition it is possible to show that the matrix $A$ is non-degenerated. The proof is given in Appendix A. Before stating the main theorem of this paper, we emphasize that the objective system is a physical model of centimeter world, whose link lengths, radius of finger-end sphere, and object width are of order of centimeter as shown in Table 1. Then, inertia moments of finger links and the object are of $O\left(10^{-5}\right)$ to $O\left(10^{-6}\right)\left[\mathrm{kgm}^{2}\right]$ in MKS units. Under this physical circumstances, we firstly choose $f_{d}>0$ appropriately (around 0.1 $\sim 5[\mathrm{~N}])$ and then choose the angular velocity feedback gain $c\left(=c_{1}=c_{2}=c_{3}\right)$ that plays a role of damping factor of the overall system in such a way that

$$
\frac{c}{2 f_{d} r}=0.1 \sim 0.3
$$

where $r$ denotes the radius of finger-end hemisphere. This guidance of damping gain tuning was proposed in the previous paper [14] suggested from Hill's model of force/velocity characteristics of muscle shortening observed in muscle physiology [15].

Now it is possible to state:

Theorem 1. If for a given reference still state $\left(\boldsymbol{x}^{0}, 0\right) \in M_{1}$ there exists a neighborhood $N^{8}\left(r_{0}\right)$ with some $r_{0}>0$ of the state $\left(\boldsymbol{x}^{0}, 0\right)$ in $R^{8}$ such that $A$ is non-degenerated in $N^{8}\left(r_{0}\right)$, then the state $\left(\boldsymbol{x}^{0}, 0\right)$ is stable on a manifold.

Proof. First, consider a scalar quantity

$$
E=V_{0}+\alpha \dot{\boldsymbol{x}}^{\mathrm{T}} \bar{H} P \boldsymbol{e} Y / r
$$

where $\alpha>0$ is a constant and

$$
P=I_{4}-J^{+} J, \quad J^{+}=J^{\mathrm{T}}\left(J J^{\mathrm{T}}\right)^{-1} .
$$


Since $P$ is a projection matrix and two column vectors of $J$ are independent of $\boldsymbol{e}$ in $N^{8}\left(r_{0}\right)$, there exists a positive constant $\sigma_{0}>0$ such that

$$
\boldsymbol{e}^{\mathrm{T}} P \boldsymbol{e} \geq \sigma_{0}\|\boldsymbol{e}\|^{2}
$$

Note that $P J^{\mathrm{T}}=0$. Hence the time-derivative of $E$ is reduced to the following:

$$
\begin{aligned}
\dot{E}= & \dot{V}_{0}+\alpha \ddot{\boldsymbol{x}}^{\mathrm{T}} \bar{H} P \boldsymbol{e} \frac{Y}{r}+\alpha \dot{\boldsymbol{x}}^{\mathrm{T}}(\dot{\bar{H}} P+\bar{H} \dot{P}) \boldsymbol{e} \frac{Y}{r}+\alpha \dot{\boldsymbol{x}}^{\mathrm{T}} \bar{H} P \boldsymbol{e} \frac{\dot{Y}}{r} \\
= & -\dot{q}^{\mathrm{T}} C \dot{q}+\alpha \boldsymbol{\lambda}^{\mathrm{T}} A P \boldsymbol{e} \frac{Y}{r}-\alpha \dot{\boldsymbol{x}}^{\mathrm{T}}\left\{\frac{1}{2} \dot{\bar{H}}+\bar{S}^{\mathrm{T}}+\bar{C}\right\} P \boldsymbol{e} \frac{Y}{r} \\
& +\alpha \dot{\boldsymbol{x}}^{\mathrm{T}}\{(\dot{\bar{H}} P+\bar{H} \dot{P}) Y+\bar{H} P \dot{Y}\} \frac{\boldsymbol{e}}{r} \\
= & -\dot{q}^{\mathrm{T}} C \dot{q}-\alpha \boldsymbol{e}^{\mathrm{T}} P \boldsymbol{e} f_{d} Y^{2} / r-\alpha \dot{\boldsymbol{x}}^{\mathrm{T}} \bar{C} P \boldsymbol{e} \frac{Y}{r} \\
& +\alpha \dot{\boldsymbol{x}}^{\mathrm{T}}\left\{\left(\frac{1}{2} \dot{\bar{H}} P+\bar{H} \dot{P}-\bar{S}^{\mathrm{T}} P\right) Y+\bar{H} P \dot{Y}\right\} \frac{\boldsymbol{e}}{r} .
\end{aligned}
$$

Since all $\dot{\bar{H}}, \dot{P}$ and $\bar{S}$ are linear and homogeneous in $\dot{\boldsymbol{x}}$, it is possible to evaluate the last term of the right hand side in the following way

(30) $\alpha \dot{\boldsymbol{x}}\left\{\left(\frac{1}{2} \dot{\bar{H}} P+\bar{H} \dot{P}-\bar{S}^{\mathrm{T}} P\right) Y+\bar{H} P \dot{Y}\right\} \boldsymbol{e} / r \leq \alpha h_{M}\{1+|Y / r|\} \mathrm{O}\left(\|\dot{\boldsymbol{x}}\|^{2}\right)$,

where $\mathrm{O}\left(\|\dot{\boldsymbol{x}}\|^{2}\right)$ means that $\mathrm{O}\left(\|\dot{\boldsymbol{x}}\|^{2}\right) /\|\dot{\boldsymbol{x}}\|^{2}$ is of $\mathrm{O}(1)$ and $h_{M}$ stands for the maximum spectre radius of matrix $\bar{H}(q)$ over all $q$. On the other hand, it follows that

$$
-\alpha \dot{\boldsymbol{x}}^{\mathrm{T}} \bar{C} P \boldsymbol{e} r^{-1} Y \leq \frac{\alpha c}{2 r f_{d}} \dot{q}^{\mathrm{T}} C \dot{q}+\frac{\alpha f_{d} Y^{2}}{2 r} \boldsymbol{e}^{\mathrm{T}} P \boldsymbol{e} .
$$

According to the assumption of choice for $C$ and $f_{d}$, it is possible to see that

$$
\dot{E} \leq-\left(1-\frac{\alpha}{3}\right) \dot{q}^{\mathrm{T}} C \dot{q}-\frac{\alpha f_{d} Y^{2}}{2 r} \boldsymbol{e}^{\mathrm{T}} P \boldsymbol{e}+\alpha\left(h_{M} / r\right)\{|Y|+r\} \mathrm{O}\left(\|\dot{\boldsymbol{x}}\|^{2}\right) .
$$

It is also important to note that the holonomic constraint of eq.(5) yields

$$
\dot{Y}+\dot{\phi} r=0,
$$

which from eq.(4), leads to

$$
\begin{aligned}
\dot{\theta}= & \dot{q}_{1}+\dot{q}_{2}+\dot{q}_{3}-r^{-1} \dot{Y} \\
= & \dot{q}_{1}+\dot{q}_{2}+\dot{q}_{3}-r^{-1}\left(\dot{x}_{0} \sin \theta+\dot{y}_{0} \cos \theta\right) \\
& -r^{-1}\left\{\left(x_{0}-x_{m}\right) \cos \theta-\left(y_{0}-y_{m}\right) \sin \theta\right\} \dot{\theta} .
\end{aligned}
$$

Since $\left(x_{0}-x_{m}\right) \cos \theta-\left(y_{0}-y_{m}\right) \sin \theta=-(l+r)$, eq.(34) implies that

$$
\dot{\theta}=-\frac{r}{l}\left(\dot{q}_{1}+\dot{q}_{2}+\dot{q}_{3}\right)+\frac{1}{l}\left(\dot{x}_{0} \sin \theta+\dot{y}_{0} \cos \theta\right) .
$$


Since $l$ is of $O\left(l_{i}\right)$ for link length $l_{i}(i=1,2,3)$, it follows from eq.(35) that

$$
|\dot{\theta}|^{2} \leq \mathrm{O}\left(\|\dot{q}\|^{2}\right) \times 10^{1}
$$

which leads to the following equality

$$
\frac{1}{6} \dot{q}^{\mathrm{T}} C \dot{q}=\frac{c}{6}\|\dot{q}\|^{2}=\|\dot{q}\|^{2} O\left(10^{-3}\right) \geq \frac{2 \sigma_{m}}{2}\left\{\dot{q}^{\mathrm{T}} H(q) \dot{q}+I \dot{\theta}^{2}\right\}=2 \sigma_{m} K,
$$

where observations that the maximum eigenvalue of $H(q)$ is of $O\left(10^{-6}\right)$ and the inertia moment of the object $I$ is of $O\left(10^{-5}\right)$ are used. Note that the quantity $K$ defined as the half of the content of bracket \{\} in eq.(37) is the total kinetic energy of the overall system of Fig. 1 and the constant $\sigma_{m}>0$ is of $O\left(10^{1}\right)$. Hence, by setting $\alpha=2.0$ in eq.(32) and noting that $|Y|$ must remain to be of $O\left(10^{-2}\right)$ and $\left(h_{M} / r\right)$ is of $O\left(10^{-3}\right)$, eq.(32) can be reduced to

$$
\dot{E} \leq-2 \sigma_{m} K-\left(2 \boldsymbol{e}^{\mathrm{T}} P \boldsymbol{e}\right) \frac{f_{d} Y^{2}}{2 r} \leq-2 \sigma V_{0}
$$

where

$$
\sigma=\min \left\{\sigma_{m}, \inf \boldsymbol{e}^{\mathrm{T}} \boldsymbol{P} \boldsymbol{e}\right\}
$$

and the infimum inside bracket \{\} should be taken along the solution trajectory over $t \in[0, \infty)$. Since $A$ is nondegenerated in $N^{8}\left(r_{0}\right)$, there exists a positive constant $\sigma_{0}>0$ satisfying eq.(28), which means that $\sigma \geq \sigma_{0}\|\boldsymbol{e}\|^{2}$. In reality, as discussed in next section, the quantity $\boldsymbol{e}^{\mathrm{T}} \mathrm{P} \boldsymbol{e}$ remains around 1.4 during maneuvering of the finger in some example. Finally, it is important to note that eq.(26) leads to

$$
\begin{aligned}
E & =V_{0}+\alpha \dot{\boldsymbol{x}}^{\mathrm{T}} \bar{H} P \boldsymbol{e} Y / r \\
& \geq V_{0}-\frac{10 \alpha h_{M}}{f_{d} r} \dot{\boldsymbol{x}}^{\mathrm{T}} \bar{H} \dot{\boldsymbol{x}}-\frac{\alpha}{40} \boldsymbol{e}^{\mathrm{T}} P \boldsymbol{e} Y^{2} f_{d} / r \\
& \geq\left(1-\varepsilon_{0}\right) K+\left(1-\varepsilon_{1}\right) \frac{f_{d} Y^{2}}{2 r},
\end{aligned}
$$

where we set $\alpha=2$ and

$$
\varepsilon_{0}=\frac{20 h_{M}}{f_{d} r}, \quad \varepsilon_{1}=\frac{1}{10} \cdot \sup \boldsymbol{e}^{\mathrm{T}} P \boldsymbol{e} .
$$

Since $P$ is a projection matrix, it follows that $\boldsymbol{e}^{\mathrm{T}} P \boldsymbol{e} \leq \boldsymbol{e}^{\mathrm{T}} \boldsymbol{e}=4$. Note that $\varepsilon_{0}$ is less than 0.5 even in the case of $f_{d}=0.1[\mathrm{~N}]$, the smallest value for $f_{d}$. Thus, inequality (40) can be reduced to

$$
E \geq \frac{1}{2} V_{0}
$$

and similarly it follows that

$$
E \leq \frac{3}{2} V_{0}
$$


From eqs.(42), (43), and (38), it follows that

$$
\dot{E} \leq-2 \sigma V_{0} \leq-\frac{4 \sigma}{3} E
$$

which shows that

$$
V_{0}(t) \leq 2 E(t) \leq 2 E(0) e^{-(4 \sigma / 3) t} \leq 3 V_{0}(0) e^{-(4 \sigma / 3) t}
$$

This inequality shows that all velocity variables $\dot{q}_{i}(t)(i=1,2,3)$ and $\dot{\theta}(t)$ together with $Y(t)$ converge to zero exponentially in time $t$ as $t \rightarrow \infty$ and the maximum magnitude of $Y(t)$ remains small dependently on the initial value of $V_{0}(t)$, that is, $V_{0}(0)$, which proves that for a given $\varepsilon>0$ there exists $\delta_{1}(\varepsilon)>0$ such that $V_{0}(t) \leq \varepsilon^{2}$ along a solution trajectory to the closed-loop equation of eq.(23) starting from an initial state lying on $N_{4}\left(\delta_{1}(\varepsilon)\right) \cap N^{8}\left(r_{1}\right)$. The remaining proof for the existence of a fixed value $r_{1}>0$ smaller than $r_{0}$ and another quantity $\delta(\varepsilon)>0$ for an arbitrarily given $\varepsilon>0$ will be given in Appendix B.

Owing to the DOF redundancy, the still state that the solution trajectory converges to is different from the reference still state because only one single variable $Y$ is specified as $Y=0$ at $t=\infty$. By carefully examining the proof of Theorem 1 , it is also possible to show the following:

Theorem 2. Under the same assumptions as in Theorem 1, there exist numbers $\delta_{1}>0$ and $r_{1}>0$ such that the neighborhood $N_{4}\left(\delta_{1}\right) \cap N^{8}\left(r_{1}\right)$ of the reference state $\left(\boldsymbol{x}^{0}, 0\right)$ is asymptotically transferable to $M_{1} \cap N^{8}\left(r_{0}\right)$.

4. Computer Simulation Results. Computer simulations are conducted by numerically solving the closed-loop dynamics of eq.(23) under the constraints of eqs.(2) and (5), when physical parameters of the system of Fig. 1 is given as in Table 1 , initial values for position variables are set as in Table 2 , and $\dot{q}_{i}(0)=0(i=1,2,3)$ and $\dot{\theta}(0)=0$. Fig. 4 shows transient responses of $Y, \theta, f$, and $\lambda$ when the values for $f_{d}$ and $c$ are chosen as in Table 3. In this case, only the desired target values for $Y$ and $f$ are specified as $Y(\infty)=0$ and $f(\infty)=f_{d}$, but those of other variables $\theta$ and $\lambda$ are not specified. As seen in Fig. $4, Y(t)$ and $f(t)$ converge quickly to their specified target values within 0.6 second, $\lambda(t)$ converges to zero also within 0.6 second, and $\theta(t)$ does to some constant value within the same period. In this case, the quantity

$$
\xi_{0}=e^{\mathrm{T}} P \boldsymbol{e}
$$

behaves as shown in Fig. 5, which implies that the parameter $\sigma$ appearing in the exponent of exponential function of inequality (45) can be chosen as $\sigma=1.4$ in this case. Hence, eq.(45) can be reduced to

$$
V_{0}(t)=3 V_{0}(0) e^{-1.87 t}
$$


which means that $Y(t)$ converges to zero exponentially with the exponent $\exp (-0.93 t)$. This theoretical evaluation on the speed of exponential convergence is rather conservative as compared with simulation results shown in Fig. 4, but it shows the importance of quantity $\xi_{0}\left(=\boldsymbol{e}^{\mathrm{T}} P \boldsymbol{e}\right)$, which implies the grade of independence of the vector $\boldsymbol{e}$ of two vectors $(\partial R / \partial \boldsymbol{x})^{\mathrm{T}}$ and $(\partial Q / \partial \boldsymbol{x})^{\mathrm{T}}$ as shown in eq. $(22)$.

Figure 6 shows another simulation result when the sensory feedback signal of eq.(11) is used, where physical parameters and initial values of the state vector are given same as in Tables 1 and 2 and feedback gains $c, \alpha$, and $\beta$ together with desired $f_{d}$ and $\theta_{d}$ are chosen as in Table 4 . The speed of convergence is remarkably retarded though responses of both $Y$ and $\theta$ eventually converge to their desired values $Y(\infty)=0$ and $\theta(\infty)=\theta_{d}=5$ [degree] and also $f(t) \rightarrow f_{d}=0.5[\mathrm{~N}]$ after around $t=50$ second as shown in Fig. 7. In this case, the scalar function $V$ defined in eq.(14) becomes a Lyapunov function for the closed-loop dynamics composed of eqs.(12) and (13), because $V$ is positive definite in the state $(\boldsymbol{x}, \dot{\boldsymbol{x}})$ under constraints of eqs.(2) and (5), that is, $V$ is positive definite on the constraint manifold $M_{4}$ defined by eq.(15). Since the time-derivative of $V$ is non-positive as described in eq.(14), LaSalle's invariance theorem [16] implies that the solution trajectory to eqs.(12) and (13) converges asymptotically to the maximum invariant set in the manifold

$$
M=\{(\boldsymbol{x}, \dot{\boldsymbol{x}}): \dot{V}=0, R=0, Q=0, \dot{R}=0, \dot{Q}=0\} .
$$

This means that $\ddot{q}(t) \rightarrow 0$ and $\ddot{\theta} \rightarrow 0$ as $t \rightarrow \infty$ and eqs.(12) and (13) are reduced to

$$
-A^{\mathrm{T}} \overline{\boldsymbol{\lambda}}+\beta l \Delta \theta \boldsymbol{e}_{\theta}=0
$$

when $t \rightarrow \infty$, where $\overline{\boldsymbol{\lambda}}=\left(\Delta f, \lambda-\beta \Delta \theta, f_{d} Y\right)^{\mathrm{T}}$ and $\boldsymbol{e}_{\theta}=(0,0,0,1)$. Since $\boldsymbol{e}_{\theta}$ is independent of all three column vectors of $A$ and $A$ is of full rank, eq.(48) implies $\bar{\lambda}(t) \rightarrow 0, Y(t) \rightarrow 0, \Delta f(t) \rightarrow 0$, and $\Delta \theta(t) \rightarrow 0$ as $t \rightarrow \infty$. In other words, the stability problem in this case is reduced to an ordinary problem of stability in the sense of Lyapunov. However, LaSalle's invariance theorem does not present any information about the speed of convergence. In order to see the convergence speed in this case, it is important to analyze a scalar quantity described as

$$
\bar{E}=V+\alpha_{0} \dot{\boldsymbol{x}}^{\mathrm{T}} \bar{H} P_{e} e Y / r+\alpha_{1} \dot{\boldsymbol{x}}^{\mathrm{T}} \bar{H} P_{\theta} \boldsymbol{e}_{\theta} \Delta \theta,
$$

where

$$
\begin{aligned}
& P_{\theta}=I_{4}-A^{+} A, \quad A^{+}=A^{\mathrm{T}}\left(A A^{\mathrm{T}}\right)^{-1} \\
& P_{e}=I_{4}-A_{\theta}^{+} A_{\theta}, \quad A_{\theta}^{+}=A_{\theta}^{\mathrm{T}}\left(A_{\theta} A_{\theta}^{\mathrm{T}}\right)^{-1} \\
& A_{\theta}=\left(\left(\frac{\partial R}{\partial \boldsymbol{x}}\right)^{\mathrm{T}},\left(\frac{\partial Q}{\partial \boldsymbol{x}}\right)^{\mathrm{T}},-\boldsymbol{e}_{\theta}\right)^{\mathrm{T}} .
\end{aligned}
$$

Note that

$$
P_{\theta} A^{\mathrm{T}}=0, \quad P_{e} A_{\theta}^{\mathrm{T}}=0
$$


and, in particular, $P_{\theta} \boldsymbol{e}=0$ and $P_{e} \boldsymbol{e}_{\theta}=0$. Then, similar to the derivation of inequality (32), it is possible to obtain

$$
\begin{aligned}
\frac{\mathrm{d}}{\mathrm{d} t} \bar{E}= & -\left(1-\frac{\alpha_{0}+\alpha_{1}}{3}\right) \dot{q}^{\mathrm{T}} C \dot{q}-\frac{\alpha_{0} f_{d} Y^{2}}{2 r} \boldsymbol{e}^{\mathrm{T}} P_{e} \boldsymbol{e}-\frac{\alpha_{1} l \beta \Delta \theta^{2}}{2} \boldsymbol{e}_{\theta}^{\mathrm{T}} P_{\theta} \boldsymbol{e}_{\theta} \\
& -\alpha_{1} l \alpha \dot{\theta}^{2}+\left\{\frac{\alpha_{0} h_{M}}{r}(|Y|+r)+\alpha_{1} h_{M}(|\Delta \theta|+1)\right\} O\left(\|\dot{\boldsymbol{x}}\|^{2}\right) .
\end{aligned}
$$

Similar to the derivation of eqs.(42) and (43), it is also possible to show

$$
\frac{1}{2} V<\bar{E}<\frac{3}{2} V
$$

provided that $\alpha_{0}$ and $\alpha_{1}$ are set as $\alpha_{0}=\alpha_{1}=1.0$ and $\beta$ is chosen as in Table 4 . Then, by defining

$$
\begin{gathered}
\xi_{e}=\boldsymbol{e}^{\mathrm{T}} P_{e} \boldsymbol{e}, \quad \xi_{\theta}=\boldsymbol{e}_{\theta}^{\mathrm{T}} P_{\theta} \boldsymbol{e}_{\theta} \\
\sigma=\min \left\{\inf \xi_{e}, \inf \xi_{\theta}\right\},
\end{gathered}
$$

where both 'inf's inside bracket \{\} are taken along the solution trajectory to the closed-loop dynamics of eqs.(12) and (13), it is possible to show

$$
V(t) \leq 3 V(0) e^{-(2 \sigma / 3) t} .
$$

Thus, the exponent parameter $\sigma$ signifies the grade of exponential convergence. In Fig. 8, transient behaviours of $\xi_{e}$ and $\xi_{\theta}$ are shown. Both $\xi_{e}$ and $\xi_{\theta}$ converge to some constant values after 50 second respectively. Since $\xi_{e}$ is fairly smaller than $\xi_{0}$ as compared with Fig. 5, it is possible to understand why the response of $Y$ in the case of feedback signal of eq.(11) is remarkably retarded in comparison with that of $Y$ in the case of eq.(16). This shows that, if the number of physical variables necessary and sufficient for description of a desired target task is less than the total DOFs, then the convergence of its solution trajectory can be speeded up. In other words, surplus DOFs of robotic systems may enhance dexterity in execution of tasks.

5. Redundancy Resolution in Case of Stable Grasping by a Pair of Multi-DOF Fingers. Next consider a problem of redundancy resolution for the dynamics of planar motion of a pair of multi-DOF robot fingers with hemi-spherical finger ends contacting with a rigid object with parallel surfaces and rolling on object surfaces (see Fig. 9). As already shown in the previous papers [9]-[10] or reasonably predicted from the discussion in derivation of eqs.(7) and (8), the overall dynamics of motion of the system are described as follows:

$$
\begin{aligned}
H_{i} \ddot{q}_{i}+\left\{\frac{1}{2} \dot{H}_{i}+S_{i}\right\} \dot{q}_{i}+(-1)^{i-1} J_{0 i}^{\mathrm{T}}\left(\begin{array}{c}
\cos \theta \\
-\sin \theta
\end{array}\right) f_{i} \\
\quad+\lambda_{i}\left\{r_{i}\left(\begin{array}{c}
1 \\
1
\end{array}\right)-J_{0 i}^{\mathrm{T}}\left(\begin{array}{c}
\sin \theta \\
\cos \theta
\end{array}\right)\right\}=u_{i}, i=1,2
\end{aligned}
$$




$$
\left\{\begin{array}{l}
M \ddot{x}-\left(f_{1}-f_{2}\right) \cos \theta+\left(\lambda_{1}+\lambda_{2}\right) \sin \theta=0 \\
M \ddot{y}+\left(f_{1}-f_{2}\right) \sin \theta+\left(\lambda_{1}+\lambda_{2}\right) \cos \theta=0 \\
I \ddot{\theta}-f_{1} Y_{1}+f_{2} Y_{2}+l_{1} \lambda_{1}-l_{2} \lambda_{2}=0
\end{array}\right.
$$

where $J_{0 i}$ denotes the Jacobian matrix of $\left(x_{0 i}, y_{0 i}\right)$ with respect to $q_{i}=\left(q_{i 1}, q_{i 2}\right)^{\mathrm{T}}$ for $i=1,2$ and $M$ and $I$ denote the mass and inertia moment of the object. It should be noted that the inertia matrix $H_{i}$ for finger $i$ depends on the position vector $q_{i}$ and hence $\dot{H}_{i}$ and $S_{i}$ depend on both $q_{i}$ and $\dot{q}_{i}$. However, it should be remarked that $S_{i}\left(q_{i}, \dot{q}_{i}\right)$ is linear and homogeneous in $\dot{q}_{i}$ and skew-symmetric. Hence, it follows that

$$
\dot{q}_{i}^{\mathrm{T}}\left[H_{i} \ddot{q}_{i}+\left\{\frac{1}{2} \dot{H}_{i}+S_{i}\right\} \dot{q}_{i}\right]=\frac{\mathrm{d}}{\mathrm{d} t} \frac{1}{2} \dot{q}_{i}^{\mathrm{T}} H_{i}\left(q_{i}\right) \dot{q}_{i}, \quad i=1,2 .
$$

Now consider the sensory feedback signal

$$
u_{s i}=-c_{i} \dot{q}_{i}-(-1)^{i} J_{0 i}^{\mathrm{T}}\left(\begin{array}{c}
\cos \theta \\
-\sin \theta
\end{array}\right) f_{d}+(-1)^{i} \frac{r_{i} f_{d}}{r_{1}+r_{2}}\left(Y_{1}-Y_{2}\right)\left(\begin{array}{l}
1 \\
1
\end{array}\right),
$$

where $c_{i}>0$ and $Y_{1}-Y_{2}$ can be calculated in real-time on the basis of measured data on $\theta(t)$ and $q_{i}(t)(i=1,2)$ as follows:

$$
Y_{1}-Y_{2}=\left(x_{01}-x_{02}\right) \sin \theta+\left(y_{01}-y_{02}\right) \cos \theta .
$$

Substituting eq.(62) into eq.(59) by setting $u_{i}=u_{s i}$ yield

$$
\begin{aligned}
H_{i} \ddot{q}_{i}+\left\{\frac{1}{2} \dot{H}_{i}+\right. & \left.S_{i}+c_{i} I_{2}\right\} \dot{q}_{i}-\left(\frac{\partial Q}{\partial q_{i}}\right)^{\mathrm{T}} \Delta f_{i}-\left(\frac{\partial R}{\partial q_{i}}\right) \lambda_{i} \\
& -(-1)^{i} \frac{r_{i} f_{d}}{r_{1}+r_{2}}\left(Y_{1}-Y_{2}\right)\left(\begin{array}{c}
1 \\
1
\end{array}\right)=0, \quad i=1,2,
\end{aligned}
$$

where

$$
\left\{\begin{array}{l}
Q=f_{1} Q_{1}+f_{2} Q_{2}, \\
Q_{i}=(-1)^{i}\left\{\left(x_{0 i}-x\right) \cos \theta-\left(y_{0 i}-y\right) \sin \theta\right\}-r_{i}-l_{i}=0 \\
R=\lambda_{1} R_{1}+\lambda_{2} R_{2}, \quad R_{i}=Y_{i}-c_{0 i}+r_{i}\left\{\pi-(-1)^{i} \theta-q_{i 1}-q_{i 2}\right\}=0 \\
\left(\frac{\partial Q}{\partial q_{i}}\right)^{\mathrm{T}}=(-1)^{i} J_{0 i}^{\mathrm{T}}\left(\begin{array}{c}
\cos \theta \\
-\sin \theta
\end{array}\right), \quad i=1,2 \\
\left(\frac{\partial R}{\partial q_{i}}\right)^{\mathrm{T}}=J_{0 i}^{\mathrm{T}}\left(\begin{array}{c}
\sin \theta \\
\cos \theta
\end{array}\right)-r_{i}\left(\begin{array}{c}
1 \\
1
\end{array}\right), \quad i=1,2 .
\end{array}\right.
$$

At this stage it is important to note that the first two equations of object dynamics described by eq.(60) are expressed on the basis of physical unit of force, that is, their numerical values are based on the unit $[\mathrm{N}]$, but the last equation of eq.(60) is based on the physical unit of torque, that is, $[\mathrm{Nm}]$. Therefore, the numerical value for the 
object mass $M$ is unbalanced in comparison with the object inertia moment $I$ in the centimeter world. In order that the coefficients of acceleration terms $(\ddot{x}, \ddot{y}, \ddot{\theta})$ are reasonably comparable to each other, it is convenient to rewrite the equation (60) into

$$
\left\{\begin{array}{l}
\bar{M} \ddot{\bar{x}}-\left(\Delta f_{1}-\Delta f_{2}\right) r \cos \theta+\left(\lambda_{1}+\lambda_{2}\right) r \sin \theta=0 \\
\bar{M} \ddot{\bar{y}}+\left(\Delta f_{1}-\Delta f_{2}\right) r \sin \theta+\left(\lambda_{1}+\lambda_{2}\right) r \cos \theta=0 \\
I \ddot{\theta}-\Delta f_{1} Y_{1}+\Delta f_{2} Y_{2}+l_{1} \lambda_{1}-l_{2} \lambda_{2}-f_{d}\left(Y_{1}-Y_{2}\right)=0
\end{array}\right.
$$

where

$$
r=10^{-2}, \quad \bar{M}=r^{2} M, \quad \bar{x}=r^{-1} x, \quad \bar{y}=r^{-1} y .
$$

Finally, the closed-loop dynamics of the overall system are expressed as

$$
H \ddot{\boldsymbol{x}}+\left\{\frac{1}{2} \dot{H}+S+C\right\} \dot{\boldsymbol{x}}-J^{\mathrm{T}} \Delta \boldsymbol{\lambda}-f_{d}\left(Y_{1}-Y_{2}\right) \boldsymbol{e}=0,
$$

where $H_{0}=\operatorname{diag}(\bar{M}, \bar{M}, I), \boldsymbol{x}=\left(q_{1}^{\mathrm{T}}, q_{2}^{\mathrm{T}}, \bar{x}, \bar{y}, \theta\right)^{\mathrm{T}}$, and

$$
\begin{aligned}
& H=\left(\begin{array}{ccc}
H_{1}\left(q_{1}\right) & 0 & 0 \\
0 & H_{2}\left(q_{2}\right) & 0 \\
0 & 0 & H_{0}
\end{array}\right) \\
& S=\left(\begin{array}{ccc}
S_{1} & 0 & 0 \\
0 & S_{2} & 0 \\
0 & 0 & 0
\end{array}\right), C=\left(\begin{array}{ccc}
c_{1} I_{2} & 0 & 0 \\
0 & c_{2} I_{2} & 0 \\
0 & 0 & 0
\end{array}\right) \\
& \left\{\begin{array}{l}
\Delta \boldsymbol{\lambda}=\left(\Delta f_{1}, \Delta f_{2}, \lambda_{1}, \lambda_{2}\right) \\
\boldsymbol{e}=\left(\frac{-r_{1}}{r_{1}+r_{2}}, \frac{-r_{1}}{r_{1}+r_{2}}, \frac{r_{2}}{r_{1}+r_{2}}, \frac{r_{2}}{r_{1}+r_{2}}, 0,0,1\right)^{\mathrm{T}}
\end{array}\right. \\
& J^{\mathrm{T}}=\left(\begin{array}{cccc}
\left(\frac{\partial Q}{\partial q_{1}}\right)^{\mathrm{T}} & 0 & \left(\frac{\partial R}{\partial q_{1}}\right)^{\mathrm{T}} & 0 \\
0 & \left(\frac{\partial Q}{\partial q_{2}}\right)^{\mathrm{T}} & 0 & \left(\frac{\partial R}{\partial q_{2}}\right)^{\mathrm{T}} \\
r \cos \theta & -r \cos \theta & -r \sin \theta & -r \sin \theta \\
-r \sin \theta & r \sin \theta & -r \cos \theta & -r \cos \theta \\
Y_{1} & -Y_{2} & -l_{1} & l_{2}
\end{array}\right) .
\end{aligned}
$$

It is easy to check that taking inner product of eq.(68) with $\dot{\boldsymbol{x}}$ gives rise to

$$
\left\{\begin{aligned}
\frac{\mathrm{d}}{\mathrm{d} t} W_{0} & =-\sum_{i=1,2} c_{i}\left\|\dot{q}_{i}\right\|^{2} \\
W_{0} & =K+\frac{f_{d}}{2\left(r_{1}+r_{2}\right)}\left(Y_{1}-Y_{2}\right)^{2}
\end{aligned}\right.
$$


where $K$ is the total kinetic energy of the system, i.e.,

$$
K=\sum_{i=1,2} \frac{1}{2} \dot{q}_{i}^{\mathrm{T}} H_{i}\left(q_{i}\right) \dot{q}_{i}+\frac{1}{2}\left\{\bar{M} \dot{\bar{x}}^{2}+\bar{M} \dot{\bar{y}}^{2}+I \dot{\theta}^{2}\right\}
$$

Description of stable grasping corresponds to the 2-dimensional manifold

$$
M_{2}=\left\{(\boldsymbol{x}, \dot{\boldsymbol{x}}): Q_{i}=0, R_{i}=0(i=1,2), Y_{1}-Y_{2}=0, \dot{\boldsymbol{x}}=0\right\}
$$

and any solution trajectory to the closed-loop dynamics of eq.(68) lies on the 6dimensional manifold

$$
M_{6}=\left\{(\boldsymbol{x}, \dot{\boldsymbol{x}}): Q_{i}=0, R_{i}=0, \dot{Q}_{i}=0, \dot{R}_{i}=0(i=1,2)\right\} .
$$

Now, consider a reference state $\left(\boldsymbol{x}^{0}, \dot{\boldsymbol{x}}=0\right) \in M_{2}$ and assume that in a neighborhood of $\left(\boldsymbol{x}^{0}, 0\right)$ with radius $\eta_{0}$ defined by

$$
N^{14}\left(\eta_{0}\right)=\left\{(\boldsymbol{x}, \dot{\boldsymbol{x}}): \frac{1}{2} \dot{\boldsymbol{x}}^{\mathrm{T}} H \dot{\boldsymbol{x}}+\frac{1}{2} \Delta \boldsymbol{x}^{\mathrm{T}} H \Delta \boldsymbol{x}<\eta_{0}^{2}\right\} ;
$$

all column vectors of $J^{\mathrm{T}}$ together with $\boldsymbol{e}$ and $\boldsymbol{e}_{\theta}=(0,0,0,0,0,0,1)^{\mathrm{T}}$ are independent to each other. Then, similar to the definition of $N_{4}(\varepsilon)$ of eq.(21), it is possible to define

$$
N_{6}(\varepsilon)=\left\{(\boldsymbol{x}, \dot{\boldsymbol{x}}): Q_{i}=0, R_{i}=0, \dot{Q}_{i}=0, \dot{R}_{i}=0(i=1,2), W_{0} \leq \varepsilon^{2}\right\} .
$$

Further, it is possible to define the concepts of stability on a manifold and asymptotic transferability to a manifold in a similar way as discussed in section 3. At this stage, it is important to remark that concerned fingers and object are of centimeter size and therefore their physical parameters are likely as given in Table 5. Now it is possible to show the following result:

Theorem 3. Under the choice of damping gains as $c_{1}=c_{2}=c>0$ so that $c / 2 f_{d}\left(r_{1}+r_{2}\right)=0.1 \sim 0.3$, the concerned reference state $\left(\boldsymbol{x}^{0}, 0\right)$ is stable on a manifold. Moreover, their exist two positive numbers $\delta_{1}$ and $\eta_{1}$ such that the neighborhood $N_{6}\left(\delta_{1}\right) \cap N^{14}\left(\eta_{1}\right)$ of $\left(\boldsymbol{x}^{0}, 0\right)$ is transferable to the manifold of $M_{2} \cap N^{14}\left(\eta_{0}\right)$.

The proof is similar to that of Theorem 1. In this case, the scalar function $W_{0}$ is neither positive definite in the state space $R^{14}$ nor on the constraint manifold $M_{6}$ defined in eq.(75). Nevertheless, $W_{0}$ plays a crucial role in the proof of Theorem 3. Since $W_{0}$ is non-negative and its time-derivative is non-positive as shown in eq.(72), $W_{0}$ is non-increasing with increase of $t$. This shows that, for an arbitrarily given $\varepsilon>0$, any solution trajectory to the closed-loop dynamics of eq.(68) start from an arbitrarily given initial state $(\boldsymbol{x}(0), \dot{\boldsymbol{x}}(0))$ lying on $N_{6}(\varepsilon) \cap N^{14}\left(\eta_{0}\right)$ remains also on $N_{6}(\varepsilon)$. The problem is whether the trajectory also remains in $N^{14}\left(\eta_{0}\right)$. To show the existence of $\delta(\varepsilon)>0$ and $\eta_{1}>0$ such that the solution trajectory starting from an 
initial state on $N_{6}(\delta(\varepsilon)) \cap N^{14}\left(\eta_{1}\right)$ remains not only on $N_{6}(\varepsilon)$ but also in $N^{14}\left(\eta_{0}\right)$, it is necessary to introduce the quantity

$$
F=W_{0}+\alpha \dot{\boldsymbol{x}}^{\mathrm{T}} H \operatorname{Pe}\left(Y_{1}-Y_{2}\right) /\left(r_{1}+r_{2}\right),
$$

where

$$
P=I_{7}-J^{+} J, \quad J^{+}=J^{\mathrm{T}}\left(J J^{\mathrm{T}}\right)^{-1} .
$$

Then, by virtue of the property of inertia matrix $H$ whose maximum eigenvalue $h_{M}$ over all $\boldsymbol{x}$ is of $O\left(10^{-5}\right)$ as remarked in derivation of eq.(66) by introduction of the scale factor $r\left(=10^{-2}\right)$, it is possible to show that, similar to eqs.(42) and (43),

$$
\frac{3}{2} W_{0} \geq F \geq \frac{1}{2} W_{0}
$$

if $\alpha$ is set as $\alpha=2.0$ and

$$
\begin{aligned}
\frac{\mathrm{d}}{\mathrm{d} t} F \leq & -\frac{c}{3}\left\{\left\|\dot{q}_{1}\right\|^{2}+\left\|\dot{q}_{2}\right\|^{2}\right\}-\frac{f_{d}\left(Y_{1}-Y_{2}\right)^{2}}{r_{1}+r_{2}} e^{\mathrm{T}} P \boldsymbol{e} \\
& +h_{M}\left\{1+\frac{\left|Y_{1}-Y_{2}\right|}{r_{1}+r_{2}}\right\} O\left(\|\dot{\boldsymbol{x}}\|^{2}\right) .
\end{aligned}
$$

Since the damping factor $c$ is sufficiently large in comparison with $h_{M}$, the maximum eigenvalue of $H$ over all $\boldsymbol{x}$, and $|\dot{\theta}|^{2}$ is of $O\left(\left\|\dot{q}_{1}\right\|^{2}+\left\|\dot{q}_{2}\right\|^{2}\right)$ according to the constraints of $\dot{R}_{i}=0$ and $\dot{Q}_{i}=0$ for $i=1,2$, it is possible to conclude that

$$
\begin{aligned}
W_{0}(t) & \leq 2 F(t) \leq 2 F(0) e^{-(4 \sigma / 3) t} \\
& \leq 3 W_{0}(0) e^{-(4 \sigma / 3) t}
\end{aligned}
$$

where

$$
\sigma=\inf _{t \in[0, \infty)} e^{\mathrm{T}} P e
$$

This shows that the velocity vector $\dot{\boldsymbol{x}}$ and $Y_{1}-Y_{2}$ converge to zero exponentially. Further, in a similar way to Appendix B, it is possible to show the existence of $\delta(\varepsilon)$ and $\eta_{1}>0$ less than $\eta_{0}$ by choosing $W_{0}(0)$ appropriately small so that any solution starting from $N_{6}(\delta(\varepsilon)) \cap N^{18}\left(\eta_{1}\right)$ remains on $N_{6}(\varepsilon) \cap N^{18}\left(\eta_{0}\right)$, which completes the proof of Theorem 3 .

Computer simulation based on physical parameters of Table 5 and initial positions of Table 6 together with physical values for $c$ and $f_{d}$ of Table 7 was conducted, which results in transient behaviours of $Y_{1}-Y_{2}$ and $\theta$ together with $\zeta_{0}=e^{\mathrm{T}} P \boldsymbol{e}$ as shown in Fig. 10. Note that $\zeta_{0}>1.24$ and hence $Y_{1}-Y_{2}$ converges to zero exponentially with the exponent greater than $4 \sigma / 3 \approx 1.65$. 
When the desired task is to realize stable grasping and orientation control of the target angle $\theta_{d}$ concurrently, the sensory feedback

$$
\left\{\begin{array}{l}
u_{i}=u_{s i}+u_{\theta i} \\
u_{\theta i}=(-1)^{i}\left\{J_{0 i}^{\mathrm{T}}\left(\begin{array}{c}
\sin \theta \\
\cos \theta
\end{array}\right)-r_{i}\left(\begin{array}{l}
1 \\
1
\end{array}\right)\right\}(\alpha \dot{\theta}+\beta \Delta \theta)
\end{array}\right.
$$

can be used, where $\Delta \theta=\theta-\theta_{d}, \alpha>0$ and $\beta>0$ are constant. In this case, if we define

$$
\left\{\begin{array}{l}
\bar{F}=W+\alpha_{0} \dot{\boldsymbol{x}}^{\mathrm{T}} H P_{e} \boldsymbol{e}\left(Y_{1}-Y_{2}\right) /\left(r_{1}+r_{2}\right)+\alpha_{1} \dot{\boldsymbol{x}}^{\mathrm{T}} H P_{\theta} \boldsymbol{e}_{0} \Delta \theta \\
W=W_{0}+\frac{l}{2} \beta \Delta \theta^{2}
\end{array}\right.
$$

and

$$
\begin{cases}P_{\theta}=I_{7}-B^{+} B, & B^{+}=B^{\mathrm{T}}\left(B B^{\mathrm{T}}\right)^{-1} \\ P_{e}=I_{7}-B_{\theta}^{+} B_{\theta}, & B_{\theta}^{+}=B_{\theta}^{\mathrm{T}}\left(B_{\theta} B_{\theta}^{\mathrm{T}}\right)^{-1} \\ B=\left(J^{\mathrm{T}},-\boldsymbol{e}\right)^{\mathrm{T}}, & B_{\theta}=\left(J^{\mathrm{T}},-\boldsymbol{e}_{\theta}\right)^{\mathrm{T}}\end{cases}
$$

then it follows that

$$
\begin{aligned}
\frac{\mathrm{d}}{\mathrm{d} t} \bar{F} \leq & -\frac{c}{6}\left\{\left\|\dot{q}_{1}\right\|^{2}+\left\|\dot{q}_{2}\right\|^{2}\right\}-l \alpha \dot{\theta}^{2} \\
& -\frac{f_{d}\left(Y_{1}-Y_{2}\right)^{2}}{2\left(r_{1}+r_{2}\right)} \boldsymbol{e}^{\mathrm{T}} P_{e} \boldsymbol{e}-\frac{l\left(\beta \Delta \theta^{2}+\alpha \dot{\theta}^{2}\right)}{2} \boldsymbol{e}_{\theta}^{\mathrm{T}} P_{\theta} \boldsymbol{e}_{\theta} .
\end{aligned}
$$

This shows that the quantities

$$
\zeta_{e}=e^{\mathrm{T}} P_{e} e, \quad \zeta_{\theta}=e_{\theta}^{\mathrm{T}} P_{\theta} \boldsymbol{e}_{\theta}
$$

play a key role in evaluation of the speed of exponential convergence of $Y_{1}-Y_{2}$ and $\Delta \theta$. Figure 11 shows that both $Y_{1}-Y_{2}$ and $\Delta \theta$ converge to zero around at $t=10.0 \sim 15.0$ but the speed of convergence is remarkably retarded in comparison with Fig. 10. This phenomenon can be expected by comparing the quantity $\zeta_{e}$ of Fig. 11 with $\zeta_{0}$ of Fig. 10. Note that $\zeta_{e}$ is reduced to around 0.012 . which is equal to about $1 / 100$ of $\zeta_{0}$.

6. Conclusions. It has been shown in this paper that ill-posedness of inverse kinematics for robotic systems with redundant DOFs can be resolved by avoiding the use of inverse kinematics and, instead of it, finding and using a sensory feedback from the task space to the joint space via multiplication of a corresponding Jacobian transpose. Hence, there is no need for introduction of any artificial performance index for uniquely solving the inverse kinematics and any feedback term from the zero space of the Jacobian matrix $J$, that is, $\left(I-J^{+} J\right) v$. Illustrative examples are taken from robotic systems with surplus DOFs under constraints. In the case of redundant robotic systems with no constraint, the problem must be reduced to the stability proof of ordinary PD feedback from the task space through a Jacobian transpose. 


\section{REFERENCES}

[1] D. E. WhITNey, Resolved motion rate control of manipulators and human prosthesis, IEEE Trans. Man-Machine Syst., MMS-10(1969), pp. 47-53.

[2] M. Vukobratovic and M. Kircanski, A dynamic approach to nominal trajectory synthesis for redundant manipulators, IEEE Trans. Syst., Man, Cybern., SMC-14(1984), pp. 580-586.

[3] T. Yoshikawa, Manipulability of robotic mechanisms, Int. J. Robotics Res., 4-25(1985), pp. $3-9$.

[4] M. Vukobratovic And M. KirCanski, Kinematics and Trajectory Synthesis of Manipulation Robots, Springer-Verlag, Berlin, Germany, 1986.

[5] J. M. Hollerbach And K.C. Suh, Redundancy resolution of manipulators through torque optimization, IEEE J. of Robotics and Automation, RA-3-4(1987), pp. 308-316.

[6] Y. Nakamura, Advanced Robotics: Redundancy and Optimization, Addison-Wesley, Reading, MA, 1991.

[7] L. Sciavicco And B. Sicilliano, Modeling and Control of Robot Manipulators, McGraw-Hill, New York, 1996.

[8] S. Arimoto, J.-H. Bae, And K. Tahara, Dynamic stable pinching by a pair of robot fingers, Proc. of 2nd IFAC Conf. on Mechatronic Systems, Berkeley, California, USA, Dec. 9-11, 2003, pp. 731-736.

[9] S. Arimoto, K. Tahara, J.-H. Bae, and M. Yoshida, A stability theory on a manifold: concurrent realization of grasp and orientation control of an object by a pair of robot fingers, Robotica, 21(2003), pp. 163-178.

[10] S. Arimoto, M. Yoshida, J.-H. Bae, and K. Tahara, Dynamic force/torque closure of $2 D$ polygonal objects by a pair of rolling contacts and sensory-motor coordination, Journal of Robotic Systems, 20(2003), pp. 517-537.

[11] P. C. MüLler, Stability of nonlinear descriptor systems, A. Angew. Math. Mech., Vol. 76, Supplement 4 (1996), pp. 9-12.

[12] P. C. MüLler, Stability and optimal control of nonlinear descriptor systems: A survey, Appl. Math. and Comp. Sci., 8-2(1998), pp. 269-286.

[13] V. B. BAJIć, Equation of perturbed motions and stability of state and semi-state systems, Int. J. Control, 47-6(1988), pp. 1849-1860.

[14] J.-H. Bae, S. Arimoto, S. Nakamura, and R. Ozawa, Gain-tuning of sensory feedback for a multi-fingered hand based on muscle physiology, Proc. of Int. Conf. Control, Automation, and Systems, Gyeongjy, Korea, Oct. 22-25, 2003, pp. 1994-1999.

[15] L. Stark, Neurological Control Systems: Studies in Bioengineering, Plenum Press, New York, 1968.

[16] J. P. LASAlle, Some extension of Liapunov's second method, IRE Trans. Circuit Theory, CT-7(1960), pp. 520-527. 
Appendix A (Proof of $\operatorname{rank}(A)=3$ ). The $4 \times 3$ matrix $A$ defined by eq.(22) can be expressed as

$$
A=\left(\begin{array}{c}
\left(J_{0}^{\mathrm{T}}, e\right) \Lambda \\
Y,-l, 1
\end{array}\right), \quad \Lambda=\left(\begin{array}{ccc}
-\cos \theta & \sin \theta & 0 \\
\sin \theta & \cos \theta & 0 \\
0 & -r & -1
\end{array}\right)
$$

and $J_{0}^{\mathrm{T}}=\left(\left(\partial x_{0} / \partial q\right)^{\mathrm{T}},\left(\partial y_{0} / \partial q\right)^{\mathrm{T}}\right), x_{0}=-l_{1} \cos q_{1}-l_{2} \cos \left(q_{1}+q_{2}\right)-l_{3} \cos \left(q_{1}+q_{2}+q_{3}\right)$, and $y_{0}=l_{1} \sin q_{1}+l_{2} \sin \left(q_{1}+q_{2}\right)+l_{3} \cos \left(q_{1}+q_{2}+q_{3}\right)$. Then,

$$
\left\{\begin{array}{l}
\frac{\partial x_{0}}{\partial q}=\left(l_{1} s_{1}+l_{2} s_{12}+l_{2} s_{123}, l_{2} s_{12}+l_{2} s_{123}, l_{3} s_{123}\right) \\
\frac{\partial y_{0}}{\partial q}=\left(l_{1} c_{1}+l_{2} c_{12}+l_{3} c_{123}, l_{2} c_{12}+l_{3} c_{123}, l_{3} c_{123}\right)
\end{array}\right.
$$

where $s_{1}=\sin q_{1}, s_{12}=\sin \left(q_{1}+q_{2}\right)$, etc. Since $\Lambda$ is apparently nonsingular, it is easy to confirm that $A$ is nondegenerated if the three 3 -dimensional vectors $\partial x_{0} / \partial q, \partial y_{0} / \partial q$, and $e$ are independent to each other. Hence, it is important to check the cases when these three vectors become dependent. Actually, these three vectors become dependent if and only if 1) $\left.q_{1}=q_{2}=0,2\right) q_{1}=\pi / 2$ and $q_{2}=0$, or 3) $q_{2}=q_{3}=0$. The first and second cases can be excluded due to the conditions R2) and R3). The third case corresponds to the finger posture such that it is stretched straightforwardly. In this case the root of $x_{0}^{2}+y_{0}^{2}$ is equal to $l_{1}+l_{2}+l_{3}$. Hence the position $\left(x_{0}, y_{0}\right)$ can not satisfy R2) and R4) simultaneously. Hence, the third case can be also excluded. Thus, $A$ must be nondegenerated under the conditions of R1) to R4).

Appendix B. To prove the existence of $\delta_{1}(\varepsilon)>0$ and a fixed number $r_{1}>0$ smaller than $r_{0}$ such that a solution trajectory to the closed-loop dynamics of eq.(12) with $\alpha=0$ and $\beta=0$ starting from $N_{4}\left(\delta_{1}(\varepsilon)\right) \cap N^{8}\left(r_{1}\right)$ remains in $N_{4}(\varepsilon) \cap N^{8}\left(r_{0}\right)$, it is convenient to define

$$
\|\boldsymbol{x}(t)-\boldsymbol{x}(0)\|_{\bar{K}}=\left\{\frac{h_{M}}{2}(q(t)-q(0))^{\mathrm{T}}(q(t)-q(0))+\frac{1}{2} I(\theta(t)-\theta(0))^{2}\right\}^{1 / 2},
$$

where $\boldsymbol{x}=\left(q^{\mathrm{T}}, \theta\right)^{\mathrm{T}}$ and $h_{M}$ stands for the maximum eigenvalue of $H(q)$ over all $q$. Then, it is necessary to see that

$$
\begin{array}{r}
\frac{\mathrm{d}}{\mathrm{d} t}\|\boldsymbol{x}(t)-\boldsymbol{x}(0)\|_{\bar{K}}=\frac{\dot{\boldsymbol{x}}^{\mathrm{T}} H_{M}(\boldsymbol{x}(t)-\boldsymbol{x}(0))}{2\|\boldsymbol{x}(t)-\boldsymbol{x}(0)\|_{\bar{K}}} \\
\leq\left\{\frac{1}{2} \dot{\boldsymbol{x}} H_{M} \dot{\boldsymbol{x}}\right\}^{1 / 2} \leq\left(\frac{h_{M}}{h_{m}}\right)^{1 / 2}\left\|\dot{\boldsymbol{x}}_{K}\right\|,
\end{array}
$$

where $h_{m}$ stands for the minimum eigenvalue of $H(q)$ over $q, H_{m}=\operatorname{diag}\left(h_{M}, h_{M}\right.$, $\left.h_{M}, I\right)$, and

$$
\|\dot{\boldsymbol{x}}\|_{K}^{2}=\frac{1}{2} \dot{q}^{\mathrm{T}} H(q) \dot{q}+\frac{1}{2} I \dot{\theta}^{2} .
$$


Then, according to eq.(46), it follows that

$$
\|\dot{\boldsymbol{x}}\|_{K}^{2} \leq V_{0}(t) \leq 3 V_{0}(0) e^{-(4 \sigma / 3) t} .
$$

This inequality together with eq.(A-2) leads to

$$
\begin{aligned}
\|\boldsymbol{x}(t)-\boldsymbol{x}(0)\|_{K}= & \left\{\frac{1}{2}(q(t)-q(0))^{\mathrm{T}} H(q)(q(t)-q(0))+\frac{I}{2}(\theta(t)-\theta(0))^{2}\right\}^{1 / 2} \\
& \leq\|\boldsymbol{x}(t)-\boldsymbol{x}(0)\|_{\bar{K}} \leq\left(\frac{h_{M}}{h_{m}}\right)^{1 / 2} \sqrt{3 V_{0}(0)} \int_{0}^{t} e^{-(2 \sigma / 3) t} \\
& \leq\left(\frac{3 h_{M} V_{0}(0)}{h_{m}}\right)^{1 / 2} \frac{3}{2 \sigma}
\end{aligned}
$$

Thus, for an arbitrarily given $\varepsilon>0$, it is possible to choose $r_{1}=r_{0} / 3$ and set

$$
\delta_{1}(\varepsilon)=\left\{\begin{array}{ccc}
\varepsilon & \text { if } & \varepsilon<\varepsilon_{0} \\
\varepsilon_{0} & \text { if } & \varepsilon \geq \varepsilon_{0}
\end{array}\right.
$$

where

$$
\varepsilon_{0}=\frac{2 \sigma r_{0}}{9}\left(\frac{h_{m}}{3 h_{M}}\right)^{1 / 2}
$$

Then, according to eq.(A-5), $V_{0}(0) \leq \delta_{1}^{2}(\varepsilon)$ implies that

$$
\|\boldsymbol{x}(t)-\boldsymbol{x}(0)\|_{K} \leq r_{0} / 3
$$

and finally it is shown that

$$
\begin{aligned}
\|\Delta \boldsymbol{x}\|_{K}+\|\dot{\boldsymbol{x}}\|_{K} & \leq\|\boldsymbol{x}(t)-\boldsymbol{x}(0)\|_{K}+\left\|\boldsymbol{x}(0)-\boldsymbol{x}^{0}\right\|_{K}+\|\dot{\boldsymbol{x}}\|_{K} \\
& \leq \frac{r_{0}}{3}+\frac{r_{0}}{3}+\frac{r_{0}}{3}=r_{0}
\end{aligned}
$$

which shows that if $(\boldsymbol{x}(0), \dot{\boldsymbol{x}}(0))$ lies in $N_{4}\left(\delta_{1}(\varepsilon)\right) \cap N^{8}\left(r_{0} / 3\right)$ then the solution trajectory $(\boldsymbol{x}(t), \dot{\boldsymbol{x}}(t))$ starting from the above initial state $(\boldsymbol{x}(0), \dot{\boldsymbol{x}}(0))$ remains to be on $N_{4}(\varepsilon) \cap N^{14}\left(r_{0}\right)$. 


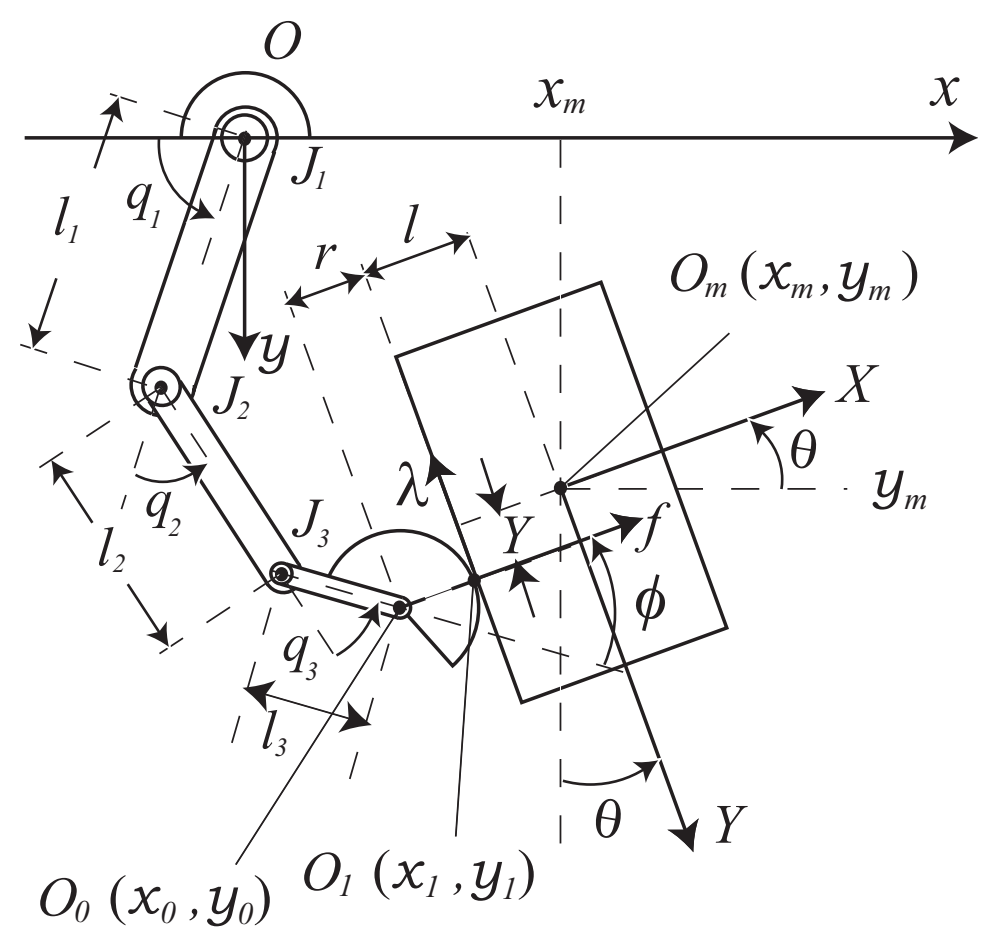

FIG. 1. Geometrical relations between a pivoted object and a 3 DOF robot finger

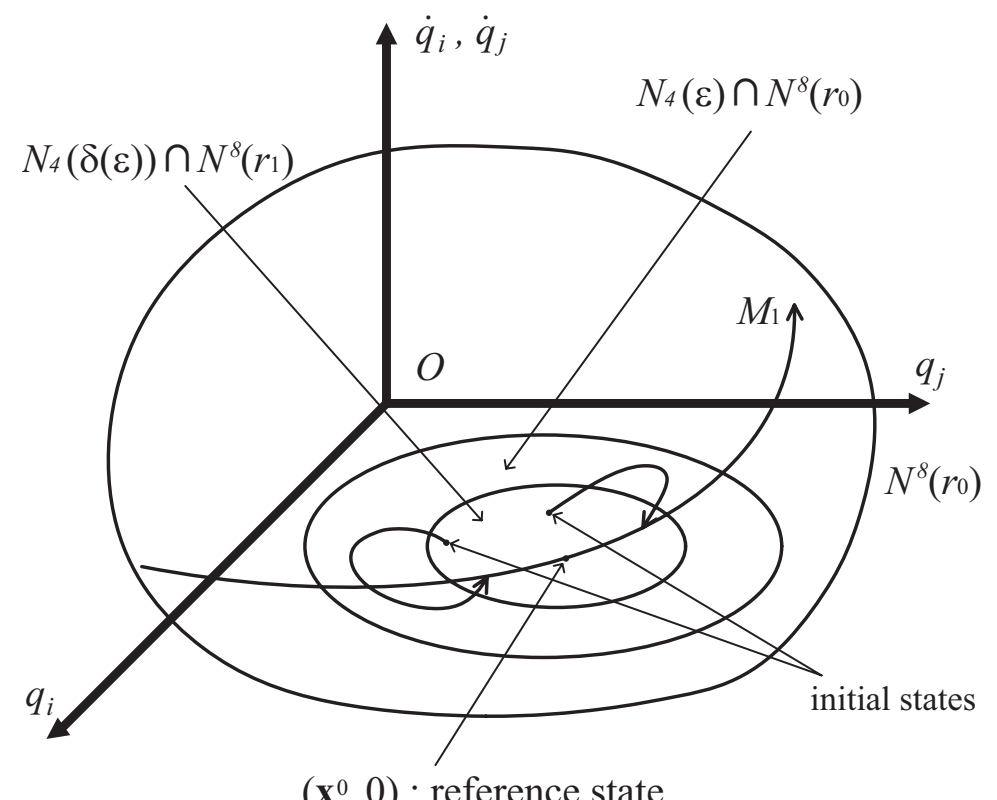

$\left(\mathbf{x}^{0}, 0\right)$ : reference state

FIG. 2. Stability on a manifold. 


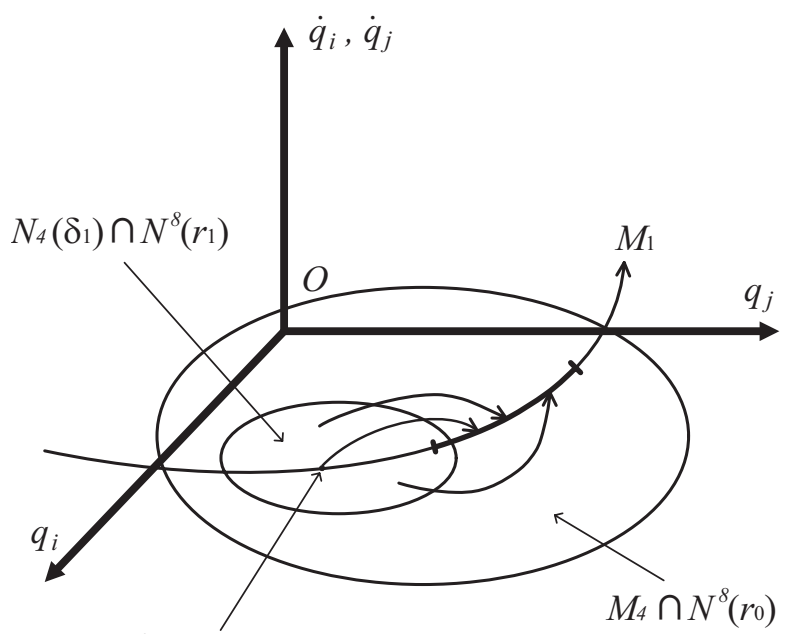

$\left(\mathbf{x}^{0}, 0\right)$ : reference steady state

FIG. 3. Any solution trajectory starting from $N_{4}\left(\delta_{1}\right) \cap N^{8}\left(r_{1}\right)$ converges asymptotically to a submanifold of $M_{1}$.
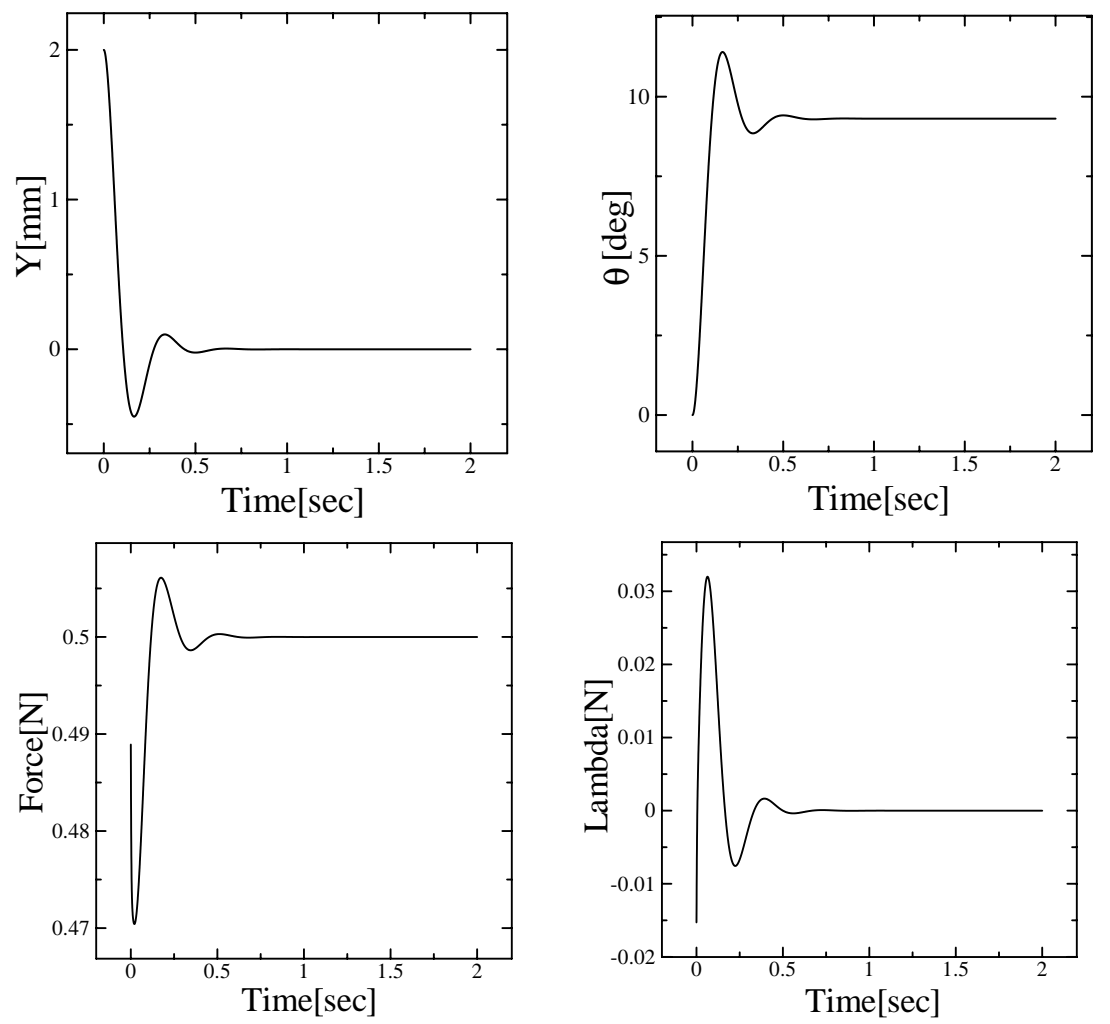

FIG. 4. Responses of $Y, \theta, f$, and $\lambda$ when the control signal of eq.(16) with gain parameters of Table 3 is used. 


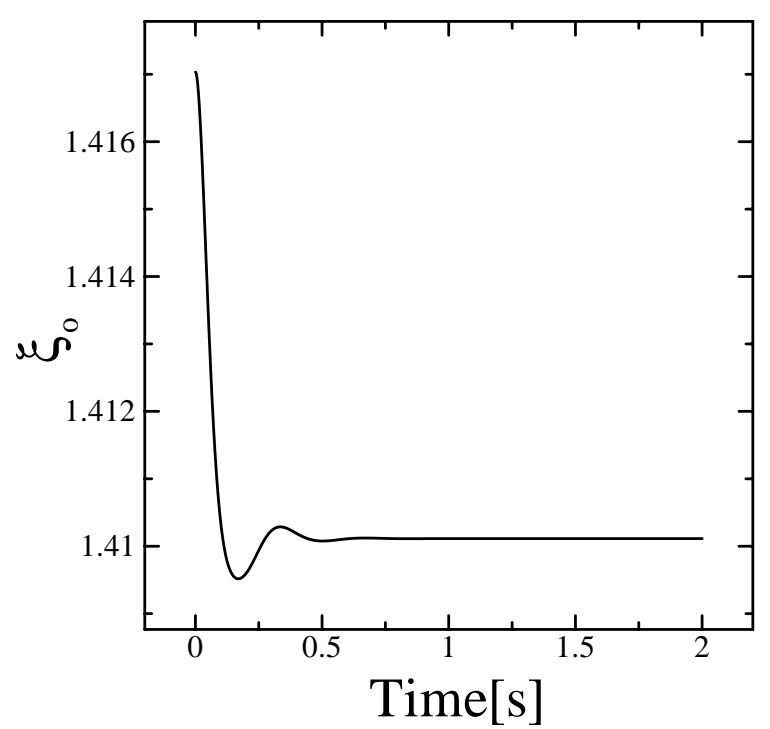

FIG. 5. Transient behaviour of the quantity $\xi_{0}$ corresponding to the case of motion of Fig. 4
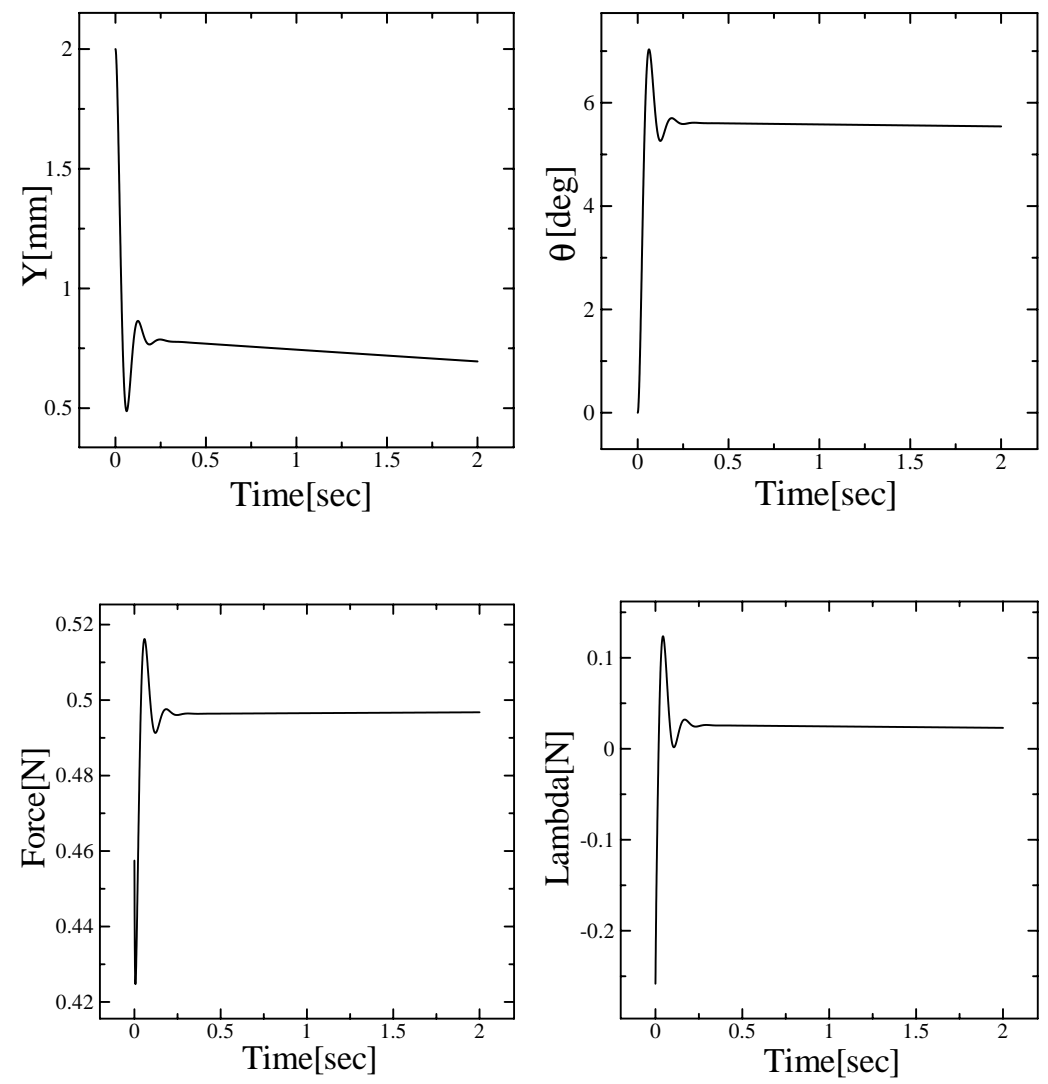

FIG. 6. Responses of $Y, \theta, f$, and $\lambda$ when the control signal of eq.(11) with parameters of Table 5 is used. 

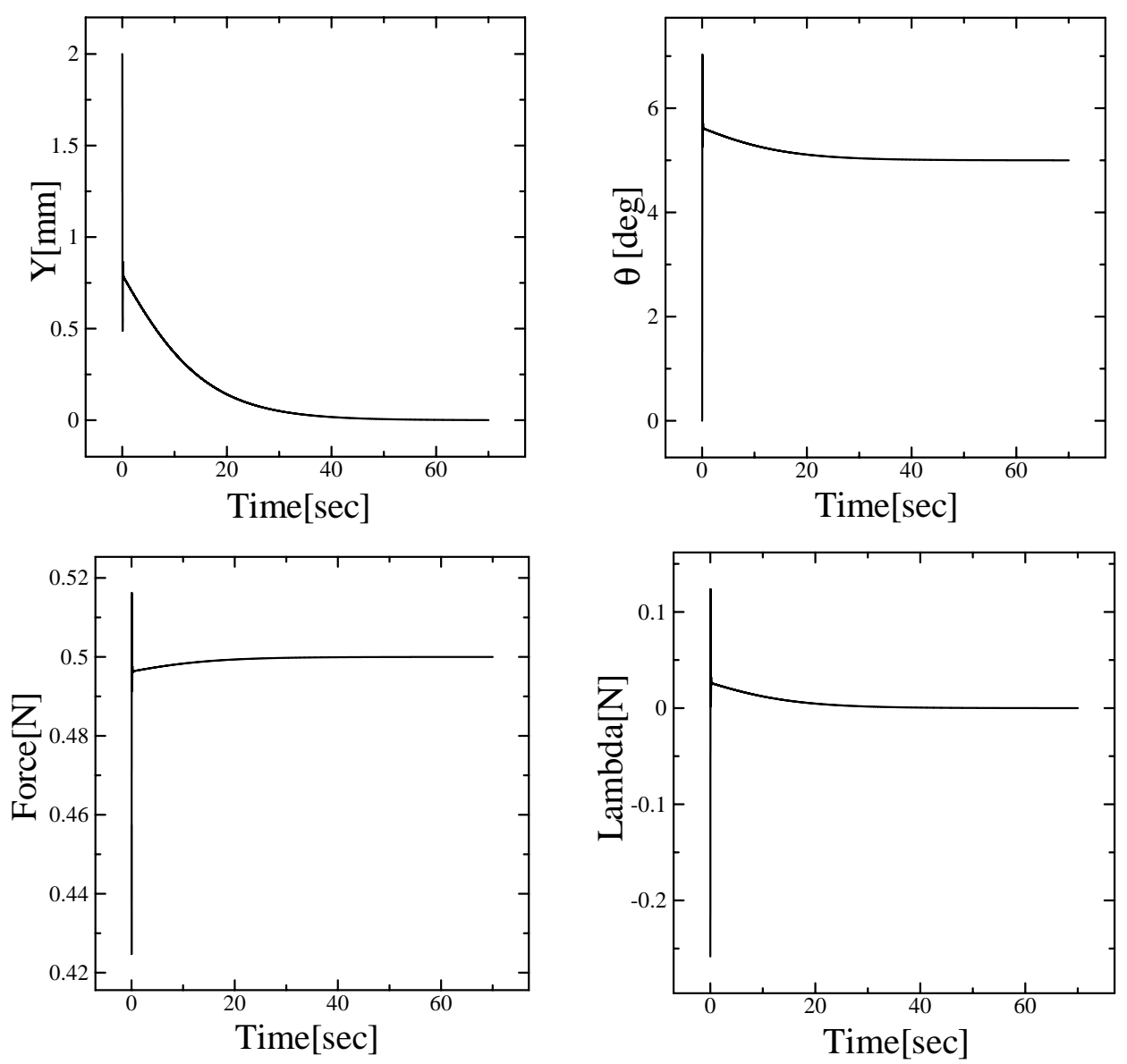

FIG. 7. The same responses as those in Fig. 4 are shown for an extended time span.
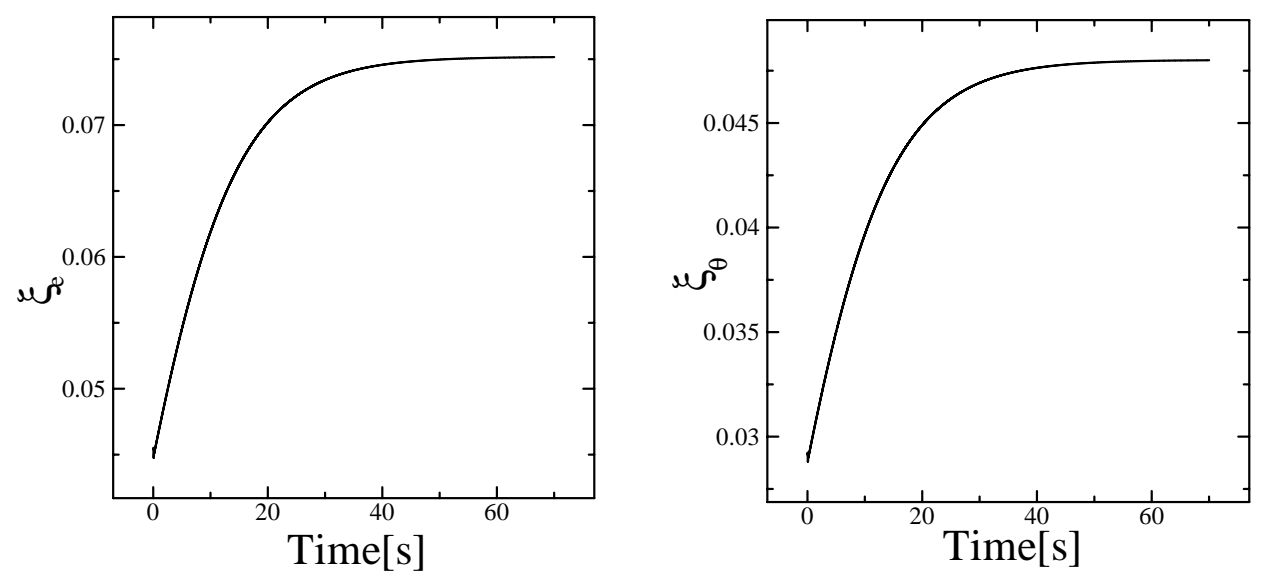

FIG. 8. Transient behaviours of $\xi_{e}$ and $\xi_{\theta}$ corresponding to the case of motion of Fig. 7 


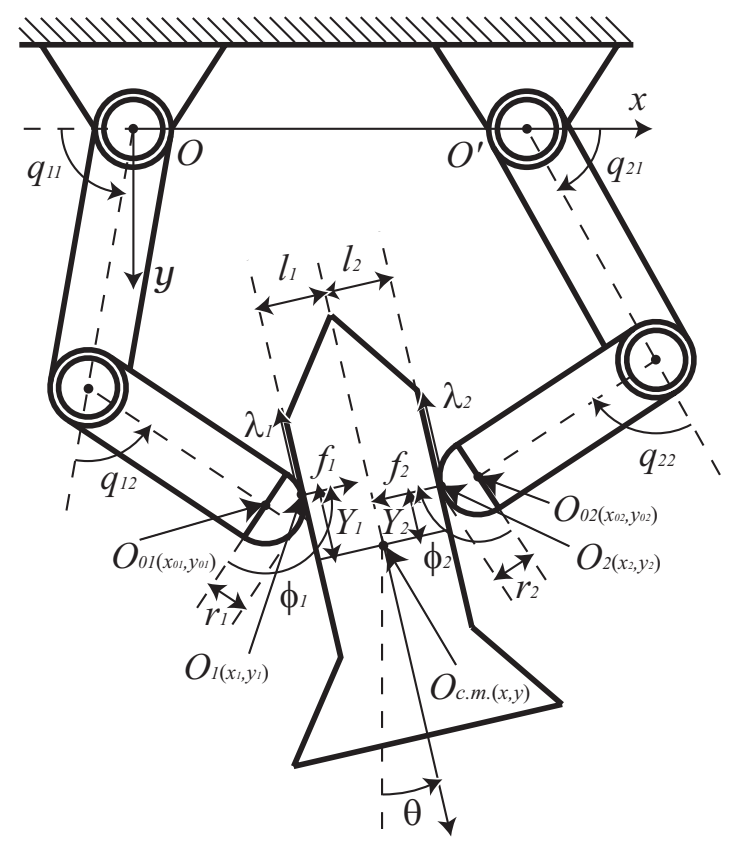

FIG. 9. A pair of dual two DOF robot fingers pinching a rigid object.
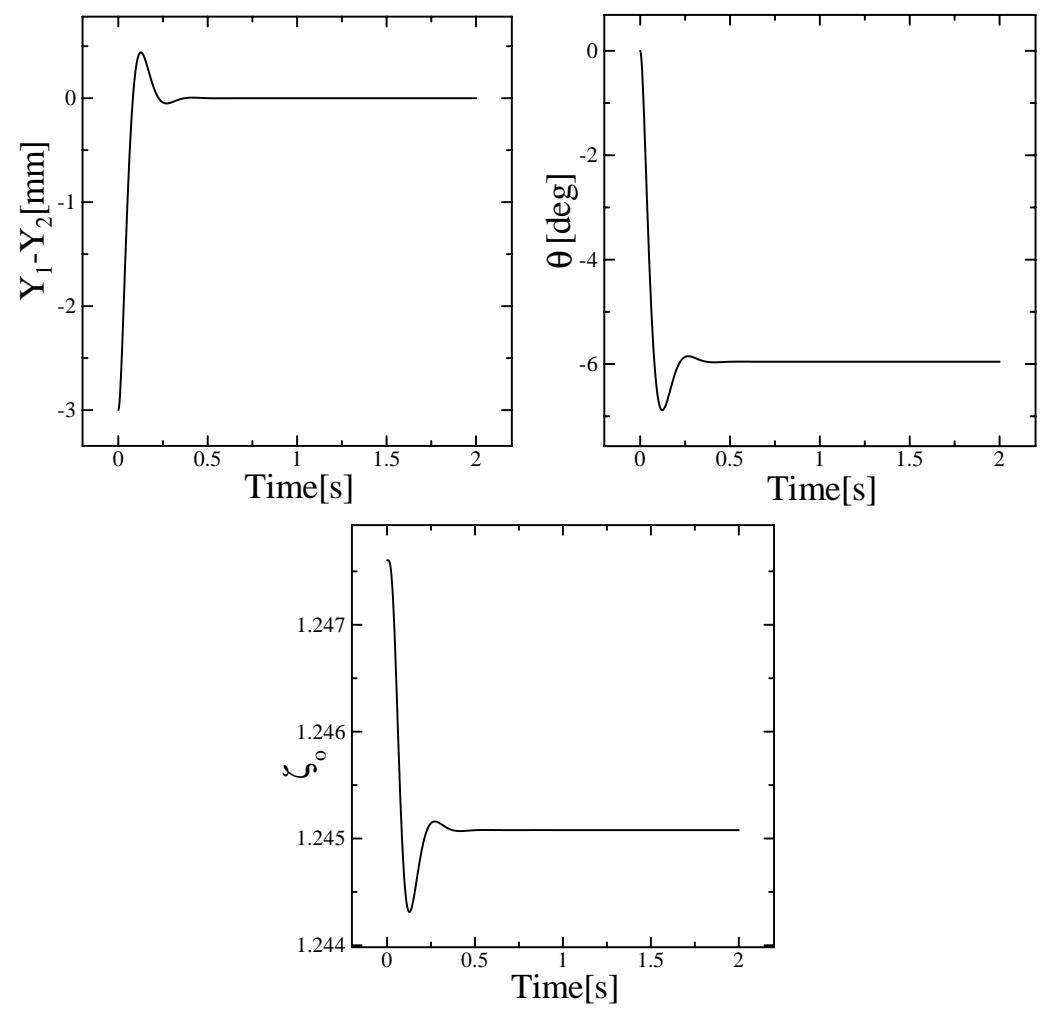

FIG. 10. Transient responses of $Y_{1}-Y_{2}, \theta$, and $\zeta_{0}$ for the closed-loop dynamics of eq.(69). 

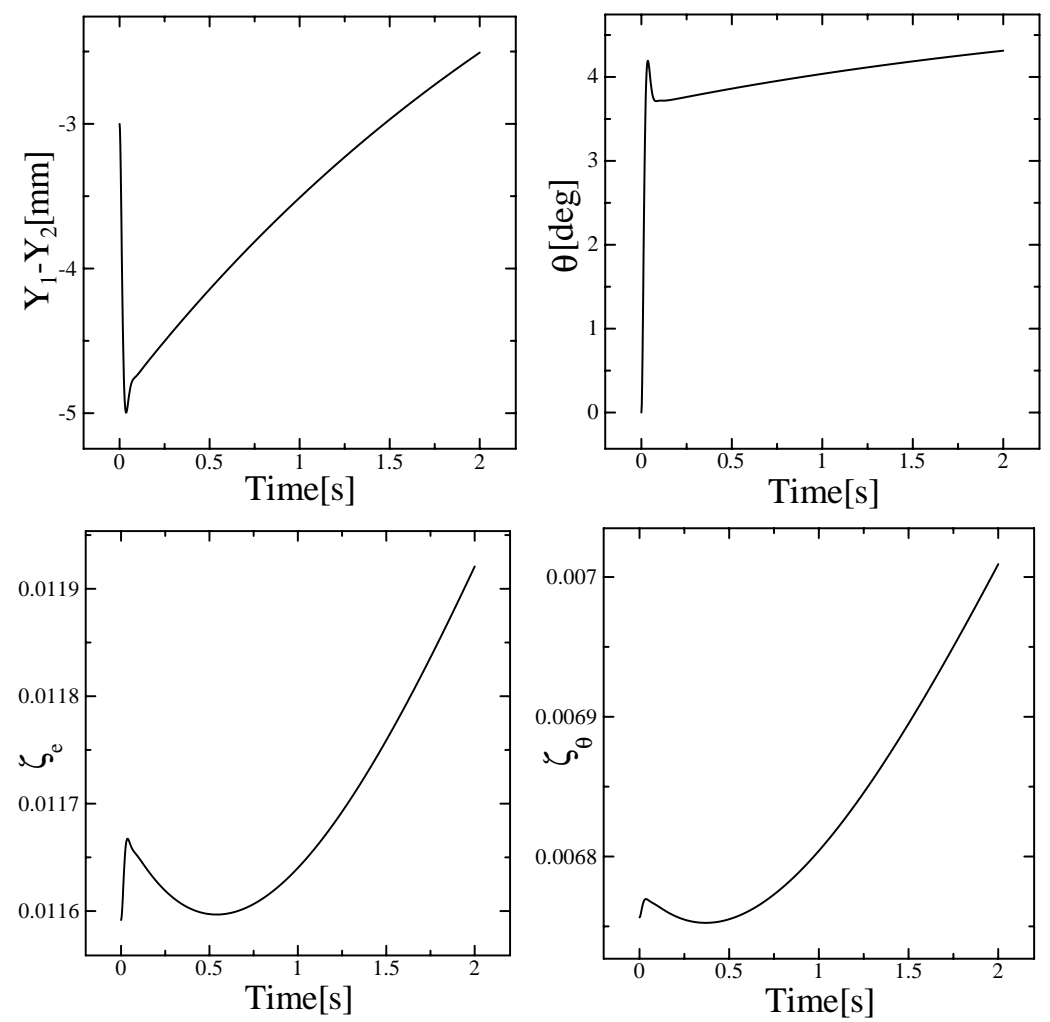

FIG. 11. Transient responses of $Y_{1}-Y_{2}, \theta, \zeta_{e}$, and $\zeta_{\theta}$ for the closed-loop dynamics of eq.(80)

TABLE 1

Physical Parameters

\begin{tabular}{c||c|c}
\hline$l_{1}$ & link length & $0.04[\mathrm{~m}]$ \\
\hline$l_{2}$ & link length & $0.04[\mathrm{~m}]$ \\
\hline$l_{3}$ & link length & $0.04[\mathrm{~m}]$ \\
\hline$m_{1}$ & link mass & $0.0450[\mathrm{~kg}]$ \\
\hline$m_{2}$ & link mass & $0.0300[\mathrm{~kg}]$ \\
\hline$m_{3}$ & link mass & $0.0150[\mathrm{~kg}]$ \\
\hline$I_{1}$ & inertia moment & $6.00 \times 10^{-6}\left[\mathrm{kgm}^{2}\right]$ \\
\hline$I_{2}$ & inertia moment & $4.00 \times 10^{-6}\left[\mathrm{kgm}^{2}\right]$ \\
\hline$I_{3}$ & inertia moment & $2.00 \times 10^{-6}\left[\mathrm{kgm}^{2}\right]$ \\
\hline$M$ & object mass & $0.05[\mathrm{~kg}]$ \\
\hline$I$ & object inertia & $1.42 \times 10^{-5}\left[\mathrm{kgm}^{2}\right]$ \\
\hline$l$ & moment & $0.015[\mathrm{~m}]$ \\
\hline$r$ & inger end radius & $0.010[\mathrm{~m}]$ \\
\hline
\end{tabular}


TABLE 2

Initial Values

\begin{tabular}{c||c}
\hline$q_{1}(0)$ & $45.0000[\mathrm{deg}]$ \\
\hline$q_{2}(0)$ & $41.7508[\mathrm{deg}]$ \\
\hline$q_{3}(0)$ & $73.0979[\mathrm{deg}]$ \\
\hline$\theta(0)$ & $0.0[\mathrm{deg}]$ \\
\hline$Y(0)$ & $2[\mathrm{~mm}]$ \\
\hline$\left(x_{m}, y_{m}\right)$ & $(0.032,0.080)[\mathrm{m}]$ \\
\hline
\end{tabular}

TABLE 3

Gains $\& 3$ desired values of force and orientation angle

\begin{tabular}{|c|c||c|c|c|}
\hline \multirow{3}{*}{ Gain } & D-gain for grasping & $c$ & 0.003 & - \\
\cline { 2 - 5 } & P-gain for posture & $\beta$ & 0 & - \\
\cline { 2 - 5 } & D-gain for posture & $\alpha$ & 0 & - \\
\hline \multirow{2}{*}{ Desired value } & force & $f_{d}$ & 0.5 & {$[\mathrm{~N}]$} \\
\cline { 2 - 5 } & angle & $\theta_{d}$ & & {$[\mathrm{deg}]$} \\
\hline
\end{tabular}

TABLE 4

Gains $\&$ desired values of force and orientation angle

\begin{tabular}{|c|c||c|c|c|}
\hline \multirow{3}{*}{ Gain } & D-gain for grasping & $c$ & 0.003 & - \\
\cline { 2 - 5 } & P-gain for posture & $\beta$ & 3 & - \\
\cline { 2 - 5 } & D-gain for posture & $\alpha$ & 0.03 & - \\
\hline \multirow{2}{*}{ Desired value } & force & $f_{d}$ & 0.5 & {$[\mathrm{~N}]$} \\
\cline { 2 - 5 } & angle & $\theta_{d}$ & 5 & {$[\mathrm{deg}]$} \\
\hline
\end{tabular}


TABLE 5

Physical Parameters

\begin{tabular}{c||c|c}
\hline$l_{11}$ & link length & $0.04[\mathrm{~m}]$ \\
\hline$l_{12}$ & link length & $0.04[\mathrm{~m}]$ \\
\hline$l_{21}$ & link length & $0.04[\mathrm{~m}]$ \\
\hline$l_{22}$ & link length & $0.04[\mathrm{~m}]$ \\
\hline$m_{11}$ & link mass & $0.04[\mathrm{~kg}]$ \\
\hline$m_{12}$ & link mass & $0.04[\mathrm{~kg}]$ \\
\hline$m_{21}$ & link mass & $0.04[\mathrm{~kg}]$ \\
\hline$m_{22}$ & link mass & $0.04[\mathrm{~kg}]$ \\
\hline$I_{i j}$ & inertia moment & $5.3333 \times 10^{-6}\left[\mathrm{kgm}^{2}\right]$ \\
\hline$M$ & object mass & $0.02[\mathrm{~kg}]$ \\
\hline$I$ & object inertia & $7.5000 \times 10^{-6}\left[\mathrm{kgm}^{2}\right]$ \\
\hline$l$ & moment & $0.030[\mathrm{~m}]$ \\
\hline$r$ & object width & $0.010[\mathrm{~m}]$ \\
\hline$L$ & finger end radius & $0.070[\mathrm{~m}]$ \\
\hline
\end{tabular}

TABLE 6

Initial Values

\begin{tabular}{c||c}
\hline$q_{11}(0)$ & $55.4469[\mathrm{deg}]$ \\
\hline$q_{12}(0)$ & $89.3554[\mathrm{deg}]$ \\
\hline$q_{21}(0)$ & $58.0387[\mathrm{deg}]$ \\
\hline$q_{22}(0)$ & $83.1619[\mathrm{deg}]$ \\
\hline$\theta(0)$ & $0.0[\mathrm{deg}]$ \\
\hline$\left(Y_{1}-Y_{2}\right)(0)$ & $-3[\mathrm{~mm}]$ \\
\hline$\left(x_{c m}, y_{c m}\right)(0)$ & $(0.035,0.060)[\mathrm{m}]$ \\
\hline
\end{tabular}


TABLE 7

Gains and Desired values

\begin{tabular}{|c|c||c|c|c|}
\hline \multirow{3}{*}{ Gain } & D-gain for grasping & $c$ & 0.003 & - \\
\cline { 2 - 5 } & P-gain for posture & $\beta$ & - & - \\
\cline { 2 - 5 } & D-gain for posture & $\alpha$ & - & - \\
\hline \multirow{2}{*}{ Desired value } & force & $f_{d}$ & 0.5 & {$[\mathrm{~N}]$} \\
\cline { 2 - 5 } & angle & $\theta_{d}$ & - & {$[\mathrm{deg}]$} \\
\hline
\end{tabular}

TABle 8

Gains and Desired values

\begin{tabular}{|c|c||c|c|c|}
\hline \multirow{3}{*}{ Gain } & D-gain for grasping & $c$ & 0.003 & - \\
\cline { 2 - 5 } & P-gain for posture & $\beta$ & 5.0 & - \\
\cline { 2 - 5 } & D-gain for posture & $\alpha$ & 0.03 & - \\
\hline \multirow{2}{*}{ Desired value } & force & $f_{d}$ & 0.5 & {$[\mathrm{~N}]$} \\
\cline { 2 - 5 } & angle & $\theta_{d}$ & 5.0 & {$[\mathrm{deg}]$} \\
\hline
\end{tabular}

